\title{
Contraction: A Unified Perspective of Correlation Decay and Zero-Freeness of 2-Spin Systems
}

\author{
Shuai Shao ${ }^{1}$ (D) Yuxin Sun ${ }^{2}$ \\ Received: 25 July 2020 / Accepted: 3 September 2021 / Published online: 26 October 2021 \\ (c) The Author(s) 2021
}

\begin{abstract}
We study the connection between the correlation decay property (more precisely, strong spatial mixing) and the zero-freeness of the partition function of 2-spin systems on graphs of bounded degree. We show that for 2-spin systems on an entire family of graphs of a given bounded degree, the contraction property that ensures correlation decay exists for certain real parameters implies the zero-freeness of the partition function and the existence of correlation decay for some corresponding complex neighborhoods. Based on this connection, we are able to extend any real parameter of which the 2-spin system on graphs of bounded degree exhibits correlation decay to its complex neighborhood where the partition function is zero-free and correlation decay still exists. We give new zero-free regions in which the edge interaction parameters and the uniform external field are all complex-valued, and we show the existence of correlation decay for such complex regions. As a consequence, we obtain approximation algorithms for computing the partition function of 2-spin systems on graphs of bounded degree for these complex parameter settings.
\end{abstract}

Keywords 2-Spin system · Correlation decay · Zero-freeness $\cdot$ Phase transition

Communicated by Eric A. Carlen.

An extended abstract of this paper appeared in the proceedings of the 47th International Colloquium on Automata, Languages and Programming (ICALP 2020), pages 96:1-96:15.

S. Shao: This work was done while the author was a graduate student in the Department of Computer Sciences, University of Wisconsin-Madison, under the support by NSF CCF-1714275.

\footnotetext{
$凶 \quad$ Shuai Shao

shuai.shao@cs.ox.ac.uk

Yuxin Sun

yxsun@cs.wisc.edu

1 Department of Computer Science, University of Oxford, Oxford, UK

2 Department of Computer Sciences, University of Wisconsin-Madison, Madison, USA
} 


\section{Introduction}

Spin systems originated from statistical physics to model interactions between neighbors on graphs. In this paper, we focus on 2-state spin (2-spin) systems. Such a system is represented as an undirected graph $G=(V, E)$, and in this way the individual entities comprising the system correspond to the vertices $V$ and their pairwise interactions correspond to the edges $E$. A configuration $\sigma$ is a mapping $\sigma: V \rightarrow\{+,-\}$ which assigns one of the two spins + and - to each vertex in $V$. Then in terms of local parameters that model the interactions of the vertices, the model induces a probability distribution (known as the Gibbs distribution) over these configurations.

In our setting, it will be convenient to parameterize these interactions in terms of the edge activities $\beta, \gamma$ that model the tendency of vertices to agree and disagree with their neighbors, and a uniform vertex activity $\lambda$ that models an external field which determines the propensity of a vertex to be spin + . The weight $w(\sigma)$ of a configuration $\sigma$ is given by $w(\sigma)=\beta^{m_{+}(\sigma)} \gamma^{m_{-}(\sigma)} \lambda^{n_{+}(\sigma)}$, where $m_{+}(\sigma)$ denotes the number of $(+,+)$ edges under the configuration $\sigma, m_{-}(\sigma)$ denotes the number of $(-,-)$ edges, and $n_{+}(\sigma)$ denotes the number of vertices assigned to spin + . The partition function $Z_{G}(\beta, \gamma, \lambda)$ of the system parameterized by $(\beta, \gamma, \lambda)$ is defined to be the sum of weights over all configurations, i.e.,

$$
Z_{G}(\beta, \gamma, \lambda)=\sum_{\sigma: V \rightarrow\{+,-\}} w(\sigma)
$$

If a 2-spin system is restricted to a family of graphs of degree bounded by a constant $\Delta$, we say such a system is $\Delta$-bounded (or bounded).

Partition functions encode rich information about the macroscopic properties of 2-spin systems. They are not only of significance in statistical physics, but also are well-studied in computer science. Computing the partition function of 2-spin systems given an input graph $G$ can be viewed as the most basic case of Counting Graph Homomorphisms (\#GH) [7,9,14,17] and Counting Constraint Satisfaction Problems (\#CSP) [6,8,10,13,15], which are two very well studied frameworks for counting problems. Many natural combinatorial problems can be formulated as 2-spin systems. For example, when $\beta=\gamma$, such a system is the famous Ising model. And when $\beta=0$ and $\gamma=1, Z_{G}(0,1, \lambda)$ is the independence polynomial of the graph $G$ (also known as the hard-core model in statistical physics); it counts the number of independent sets of the graph $G$ when $\lambda=1$.

In classical statistical mechanics the parameters $(\beta, \gamma, \lambda)$ are usually non-negative real numbers, and such 2 -spin systems are divided into the ferromagnetic case $(\beta \gamma>1)$ and the antiferromagnetic case $(\beta \gamma<1)$. The case $\beta \gamma=1$ is degenerate. When $(\beta, \gamma, \lambda)$ are non-negative numbers and they are not all zero, the partition function can be viewed as the normalizing factor of the Gibbs distribution, which is the distribution where a configuration $\sigma$ is drawn with probability $\operatorname{Pr}_{G ; \beta, \gamma, \lambda}(\sigma)=\frac{w(\sigma)}{Z_{G}(\beta, \gamma, \lambda)}$. However, it is meaningful to consider parameters of complex values. The study of the location of complex zeros of the partition function $Z_{G}(\beta, \gamma, \lambda)$ connects closely to the analyticity of the free energy $\log Z_{G}(\beta, \gamma, \lambda)$, which is a classical notion in statistical physics for defining and understanding the phenomenon of phase transitions.

One of the first and also the best known results regarding the zeros of the partition function is the Lee-Yang theorem [24] for the Ising model. This result was later extended to more general models by several people $[1,27,32,37,40]$. Another standard notion for formalizing phase transitions in 2-spin systems is correlation decay. A 2-spin system on an infinite graph such as the Bethe lattice (infinite $\Delta$-regular tree) or the lattice $Z^{d}$ exhibits correlation decay if under the Gibbs distribution, correlations between spins decay exponentially with the 
distance between them. The correlation decay property is also known as spatial mixing. ${ }^{1}$ The correlation decay property can be extended to 2-spin systems on infinite families of finite graphs.

In seminal works $[11,12]$, Dobrushin and Shlosman proved that the correlation decay property is actually equivalent to the analyticity of the free energy and the zero-freeness of the partition function for the special case of lattices. Recently, some more refined connections between them have been observed in the hard-core model [34] and the Ising model [30,35] for the setting of general graphs of bounded degree. For these models, one can extend a real interval in which correlation decay exists to its complex neighborhood where the partition function is zero-free. In particular, based on this connection, Peter and Regts solved Sokal's conjecture [44] on the zeros of the partition function of the hard-core model on graphs of bounded degree [34]. In this paper, we further study the connection between correlation decay and the zero-freeness of the partition function for 2-spin systems on general graphs of bounded degree. Specifically, we employ the standard potential method developed for the analysis of correlation decay to study the zeros of the partition function $Z_{G}(\beta, \gamma, \lambda)$ of 2-spin systems, viewed as a multivariate polynomial over these three complex parameters $(\beta, \gamma, \lambda)$.

We show that for bounded 2-spin systems, the existence of correlation decay and the zero-freeness of the partition function can be unified via a single perspective: contraction. In addition, correlation decay and the zero-freeness can be exploited to devise approximation algorithms for computing the partition function of 2-spin systems via Weitz's algorithm [47] and Barvinok's algorithm [3] respectively. Based on the connection of the two notions, we unify Weitz's algorithm and Barvinok's algorithm for bounded 2-spin systems. We also obtain new zero-free regions of the partition function of bounded 2-spin systems and new approximation algorithms for computing the partition function in these zero-free regions. Before we formally describe our results, we give some background about the approximation algorithms for the partition function of 2-spin systems.

\section{Approximation Algorithms}

For exact computation of $Z_{G}(\beta, \gamma, \lambda)$, the problem is proved to be \#P-hard for all complex valued parameters but a few very restricted trivial settings $[2,9,10]$. So the main focus is to approximate $Z_{G}(\beta, \gamma, \lambda)$. This is an area of active research, and many inspiring algorithms have been developed. The pioneering algorithm developed by Jerrum and Sinclair gives a fully polynomial-time randomized approximation scheme (FPRAS) for the ferromagnetic Ising model [22]. An FPRAS is a family of algorithms $\left\{A_{\varepsilon, \delta}\right\}$, where $A_{\varepsilon, \delta}$ is a multiplicative $(1 \pm \varepsilon)$-approximation algorithm with probability at least $1-\delta$ and running time polynomial in $1 / \varepsilon$ and $\log (1 / \delta)$ for each $\varepsilon, \delta>0$. The FPRAS in [22] is based on the Markov Chain Monte Carlo (MCMC) method which devises approximate counting algorithms via random sampling. Later, it was extended to general ferromagnetic 2 -spin systems [18,29]. The MCMC method can only handle non-negative parameters as it is based on probabilistic sampling.

A fully polynomial-time approximation scheme (FPTAS) is a family of algorithms $\left\{A_{\varepsilon}\right\}$, where $A_{\varepsilon}$ is a multiplicative $(1 \pm \varepsilon)$-approximation algorithm with running time polynomial in $1 / \varepsilon$ for each $\varepsilon>0$. The correlation decay property and the zero-freeness of the partition function can be exploited directly to devise FPTASs for 2-spin systems. The method associated with correlation decay developed by Weitz [47] was originally used to devise the FPTAS

1 There are weak spatial mixing and strong spatial mixing. Weak spatial mixing just refers to the correlation decay property; strong spatial mixing requires that correlations between spins decay exponentially with distance even when there are fixed spins (boundary conditions) close to the spins being measured. 
for the hardcore model up to the uniqueness threshold of the infinite regular tree. It turns out to be a very powerful tool for devising FPTASs for antiferromagnetic 2-spin systems $[25,26,42,48]$. Combining with hardness results $[16,43]$, an exact threshold of computational complexity transition of antiferromagnetic 2-spin systems is identified and the only remaining case is at the critical point. On the other hand, for ferromagnetic 2-spin systems, limited results $[20,48]$ have been obtained via the correlation decay method. Although correlation decay is usually analyzed in 2-spin systems of non-negative parameters, it can be adapted to complex parameters. An FPTAS was obtained for the hard-core model in the Shearer region (a disc in the complex plane) via correlation decay in [21].

Recently, a new method developed by Barvinok [3], and extended by Patel and Regts [33] is the Taylor polynomial interpolation method. This method turns complex zero-free regions of the partition function into FPTASs of corresponding complex parameters. Suppose that the partition function $Z_{G}(\beta, \gamma, \lambda)$ has no zero in a complex region containing an easy computing point, e.g., $(\beta, \gamma, \lambda)$ where $\lambda=0$. It turns out that, possibly after a change of coordinates, $\log Z_{G}(\beta, \gamma, \lambda)$ is well approximated in a slightly smaller region by a low degree Taylor polynomials which can be efficiently computed. This method connects the long-standing study of complex zeros to algorithmic studies of the partition function of physical systems. Motivated by this, more recently some complex zero-free regions have been obtained for hard-core models [5,34], Ising models [30,35], and general 2-spin systems [19].

\section{Our Contribution}

In this paper, we show that for bounded 2-spin systems, the real contraction property (Definition 2.4$)^{2}$ that ensures correlation decay exists for certain real parameters directly implies the zero-freeness and the existence of correlation decay of corresponding complex neighborhoods. Then via either Weitz's algorithm or Barvinok's algorithm, there is an FPTAS for computing the partition function of bounded 2-spin systems in these complex neighborhoods. We formally describe our main result. We use $\zeta \in \mathbb{C}^{3}$ to denote the parameter vector $(\beta, \gamma, \lambda)$. Since the case $\beta=\gamma=0$ is trivial, by symmetry we always assume $\gamma \neq 0$. For a complex vector $\mathbf{z}=\left(z_{1}, \ldots, z_{n}\right) \in \mathbb{C}^{n}$, we use $\|\mathbf{z}\|_{\infty}=\max \left\{\left|z_{1}\right|, \ldots,\left|z_{n}\right|\right\}$ to denote its infinity-norm, and $\|\mathbf{z}\|_{1}=\left|z_{1}\right|+\cdots+\left|z_{n}\right|$ to denote its 1-norm. $\left|z_{i}\right|$ denotes the standard Euclidean norm of a complex number.

Theorem 1.1 Fix $\Delta \in \mathbb{N}$. If $\zeta_{0} \in \mathbb{R}^{3}$ satisfies real contraction (see Definition 2.4) for $\Delta$, then there exists a $\delta>0$ such that for any $\zeta \in \mathbb{C}^{3}$ where $\left\|\zeta-\zeta_{0}\right\|_{\infty}<\delta$, we have

- $Z_{G}(\zeta) \neq 0$ for every graph ${ }^{3} G$ of degree at most $\Delta$;

- the $\Delta$-bounded 2-spin system specified by $\zeta$ exhibits correlation decay.

Then via either Weitz's algorithm or Barvinok's algorithm, there is an FPTAS for computing the partition function $Z_{G}(\zeta)$.

This result formally establishes the inherent connection between the existence of correlation decay and the zero-freeness of the partition function for bounded 2-spin systems via a unified perspective, contraction. The connection from the existence of correlation decay of real parameters to the zero-freeness of corresponding complex neighborhoods was already observed in the hard-core model [34] and the Ising model [30,35]. In this paper, we extend it to general 2-spin systems. Furthermore, crucially based on the zero-freeness, we also show

\footnotetext{
2 In many cases, the existence of correlation decay boils down to this property.

3 This is true even if $G$ contains some vertices pinned by a feasible configuration (Definition 2.2).
} 
the existence of correlation decay for the corresponding complex parameters. This establishes the connection from the zero-freeness of complex neighborhoods back to the existence of correlation decay of such complex regions.

For non-negative parameters of which bounded 2-spin systems are known to exhibit correlation decay $[26,48]$, we can verify that they all satisfy real contraction. Thus, Theorem 1.1 gives a very simple but general approach to extend any real parameter of which the bounded 2-spin system exhibits correlation decay to its complex neighborhood where the partition function is zero-free and correlation decay still exists. Based on this approach, we obtain new zero-free regions of the partition function of 2-spin systems. They are the first zero-free regions in which all three parameters $(\beta, \gamma, \lambda)$ are complex-valued.

We give our zero-free regions by identifying the following sets of real parameters of which bounded 2-spin systems exhibit correlation decay.

Definition 1.2 Fix integer $\Delta \geq 3$. We define the following four real correlation decay sets.

1. $\mathcal{S}_{1}^{\Delta}=\left\{\zeta \in \mathbb{R}^{3} \mid \frac{\Delta-2}{\Delta}<\sqrt{\beta \gamma}<\frac{\Delta}{\Delta-2}, \beta, \gamma>0\right.$ and $\left.\lambda \geq 0\right\}$,

2. $\mathcal{S}_{2}^{\Delta}=\left\{\zeta \in \mathbb{R}^{3} \mid \beta \gamma<1, \beta \geq 0, \gamma>0, \lambda \geq 0\right.$, and $\zeta$ is up-to- $\Delta$ unique (Definition 2.6)\},

3. $\mathcal{S}_{3}^{\Delta}=\left\{\zeta \in \mathbb{R}^{3} \mid \beta \gamma>\frac{\Delta}{\Delta-2}, \beta, \gamma>0\right.$ and $\left.0 \leq \lambda<\frac{\gamma}{t^{\Delta-1}[(\Delta-2) \beta \gamma-\Delta]}\right\}$ where $t=$ $\max \{1, \beta\}$

4. and $\mathcal{S}_{4}^{\Delta}=\left\{\zeta \in \mathbb{R}^{3} \mid \beta \gamma>\frac{\Delta}{\Delta-2}, \beta, \gamma>0\right.$ and $\left.\lambda>\frac{(\Delta-2) \beta \gamma-\Delta}{\beta r^{\Delta-1}}\right\}$ where $r=$ $\min \{1,1 / \gamma\}$.

When context is clear, we omit the superscript $\Delta$.

The set $\mathcal{S}_{1}^{\Delta}$ was given in [48] and $\mathcal{S}_{2}^{\Delta}$ was given in [26]. To our best knowledge, $\mathcal{S}_{1}^{\Delta}$ and $\mathcal{S}_{2}^{\Delta}$ cover all non-negative parameters of which bounded 2 -spin systems are known to exhibit correlation decay. The sets $\mathcal{S}_{3}^{\Delta}$ and $\mathcal{S}_{4}^{\Delta}$ are obtained in this paper ${ }^{4}$. They give new correlation decay results and hence an FPTAS for bounded ferromagnetic 2-spin systems. When $\beta<\gamma$ and $\lambda$ is sufficiently large, it is known that approximating the partition function of ferromagnetic 2-spin systems over general graphs is \#BIS-hard [29]. Our result on $\mathcal{S}_{4}^{\Delta}$ shows that there is an FPTAS for such a problem when restricted to graphs of bounded degree. When $\beta<1<\gamma$, the FPTAS obtained from $\mathcal{S}_{3}^{\Delta}$ is covered by [20].

Theorem 1.3 Fix integer $\Delta \geq 3$. For every $\zeta_{0} \in \mathcal{S}_{i}^{\Delta}(i \in[4])$, there exists a $\delta>0$ such that for any $\zeta \in \mathbb{C}^{3}$ where $\left\|\zeta-\zeta_{0}\right\|_{\infty}<\delta$, we have

- $Z_{G}(\zeta) \neq 0$ for every graph $G$ of degree at most $\Delta$;

- the $\Delta$-bounded 2-spin system specified by $\zeta$ exhibits correlation decay.

Then via either Weitz's algorithm or Barvinok's algorithm, there is an FPTAS for computing the partition function $Z_{G}(\zeta)$.

Remark The choice of $\delta$ does not depend on the size of the graph, only on $\Delta$ and $\zeta_{0}$.

\section{Organization}

This paper is organized as follows. In Sect. 2, we briefly describe Weitz's algorithm [47]. We introduce real contraction as a sufficient condition for the existence of correlation decay

\footnotetext{
4 Since we do not assume $\beta \leq \gamma$ or $\beta \geq \gamma, \mathcal{S}_{3}^{\Delta}$ and $\mathcal{S}_{4}^{\Delta}$ are essentially the same by swapping $\beta$ and $\gamma$ and replacing $\lambda$ with $1 / \lambda$. However, if one restrict to $\beta \leq \gamma$, then $\mathcal{S}_{3}^{\Delta}$ is no longer the same as $\mathcal{S}_{4}^{\Delta}$.
} 


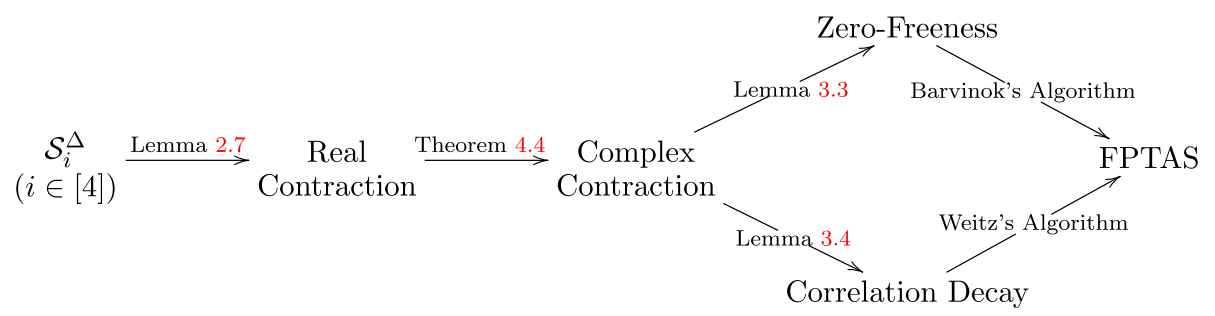

Fig. 1 The structure of our approach

of real parameters, and we show that all points in sets $\mathcal{S}_{i}^{\Delta}(i \in[4])$ satisfy it. In Sect. 3 , we briefly describe Barvinok's algorithm [3]. We introduce complex contraction as a generalization of real contraction, and we show that it gives a unified sufficient condition for both the zero-freeness of the partition function and the existence of correlation decay of complex parameters. Finally, in Sect. 4, we prove our main result that real contraction implies complex contraction. This finishes the proof of Theorem 1.3. We use the diagram (Fig. 1) to summarize our approach to establish the connection between correlation decay and the zero-freeness. We expect it to be further explored for other models.

\section{Independent Work}

After a preliminary version [39] of this manuscript was posted, we learned that based on similar ideas, Liu simplified the proofs of [34] and [30], and generalized them to antiferromagnetic Ising models $(\beta=\gamma<1)$ in chapter 3 of his Ph.D. thesis [28], where similar zero-freeness results (a complex neighborhood of $\mathcal{S}_{2}^{\Delta}$ restricted to $\beta=\gamma$ ) were obtained. We mention that by using the unique analytic continuation and the inverse function theorem, our main technical result (Theorem 4.4) is generic; it does not rely on a particularly chosen potential function. Thus, in our approach we can work with any existing potential function based argument for correlation decay even if the potential function does not have an explicit expression, for instance, the one used in [26] when $\beta \neq \gamma$. Furthermore, we mention also that based on the zero-freeness, we obtain new correlation decay results for complex parameters (Lemma 3.4), which give an FPTAS for these parameter settings via Weitz's algorithm.

\section{Weitz's Algorithm}

In this section, we describe Weitz's algorithm and introduce real contraction. We first consider positive parameters $\zeta \in \mathbb{R}_{+}^{3}$. An obvious but important fact about $\zeta$ being positive is that $Z_{G}(\zeta) \neq 0$ for any graph $G$. This is true even if $G$ contains arbitrary number of vertices pinned to spin + or - . Then, the partition function can be viewed as the normalizing factor of the Gibbs distribution.

\subsection{Notations and Definitions}

Let $\zeta \in \mathbb{R}_{+}^{3}$. We use $p_{v}(\zeta)$ to denote the marginal probability of $v$ being assigned to spin + in the Gibbs distribution, i.e., $p_{v}(\zeta)=\frac{Z_{G, v}^{+}(\zeta)}{Z_{G}(\zeta)}$, where $Z_{G, v}^{+}(\zeta)$ is the contribution to $Z_{G}(\zeta)$ 
over all configurations with $v$ being assigned to spin + . We know that $p_{v}(\zeta)$ is well-defined since $Z_{G}(\zeta) \neq 0$. (Later, we will extend the definition of $p_{v}(\zeta)$ to complex parameters $\zeta$.)

Let $\sigma_{\Lambda} \in\{0,1\}^{\Lambda}$ be a configuration of some subset $\Lambda \subseteq V$. We allow $\Lambda$ to be the empty set. We call vertices in $\Lambda$ pinned and other vertices free. We use $p_{v}^{\sigma_{\Lambda}}(\zeta)$ to denote the marginal probability of a free vertex $v(v \notin \Lambda)$ being assigned to spin + conditioning on the configuration $\sigma_{\Lambda}$ of $\Lambda$, i.e., $p_{v}^{\sigma_{\Lambda}}(\zeta)=\frac{Z_{G, v}^{\sigma_{\Lambda},+}(\zeta)}{Z_{G}^{\sigma_{\Lambda}}(\zeta)}$, where $Z_{G}^{\sigma_{\Lambda}}(\zeta)$ is the weight over all configurations where vertices in $\Lambda$ are pinned by the configuration $\sigma_{\Lambda}$, and $Z_{G, v}^{\sigma_{\Lambda},+}(\zeta)$ is the contribution to $Z_{G}^{\sigma_{\Lambda}}(\zeta)$ with $v$ being assigned to spin + . Correspondingly, we can define $Z_{G, v}^{\sigma_{\Lambda},-}(\zeta)$. Let $R_{G, v}^{\sigma_{\Lambda}}(\zeta):=\frac{Z_{G, v}^{\sigma_{\Lambda},+}(\zeta)}{Z_{G, v}^{\sigma_{\Lambda}-}(\zeta)}=\frac{p_{v}^{\sigma_{\Lambda}}(\zeta)}{1-p_{v}^{\sigma_{\Lambda}}(\zeta)}$ be the ratio between the two probabilities that the free vertex $v$ is assigned to spin + and - , while imposing some condition $\sigma_{\Lambda}$. Since $Z_{G}(\zeta) \neq 0$ for any graph $G$ with arbitrary number of pinned vertices, both $p_{v}^{\sigma_{\Lambda}}(\zeta)$ and $R_{G, v}^{\sigma_{\Lambda}}(\zeta)$ are well-defined. When context is clear, we write $p_{v}(\zeta), p_{v}^{\sigma_{\Lambda}}(\zeta)$ and $R_{G, v}^{\sigma_{\Lambda}}(\zeta)$ as $p_{v}$, $p_{v}^{\sigma_{\Lambda}}$ and $R_{G, v}^{\sigma_{\Lambda}}$ for convenience.

Note that computing the partition function of 2-spin systems is self-reducible. If one can compute $p_{v}$ for any vertex $v$, then the partition function can be computed via telescoping [23]. The goal of Weitz's algorithm is to estimate $p_{v}^{\sigma_{\Lambda}}$, which is equivalent to estimating $R_{G, v}^{\sigma_{\Lambda}}$. For the case that the graph is a tree $T, R_{T, v}^{\sigma_{\Lambda}}$ can be computed by recursion. Suppose that a free vertex $v$ has $d$ children, and $s_{1}$ of them are pinned to,$+ s_{2}$ are pinned to -, and $k$ are free $\left(s_{1}+s_{2}+k=d\right)$. We denote these $k$ free vertices by $v_{i}(i \in[k])$ and let $T_{i}$ be the corresponding subtree rooted at $v_{i}$. We use $\sigma_{\Lambda}^{i}$ to denote the configuration $\sigma_{\Lambda}$ restricted to $T_{i}$. Since all subtrees are independent, it is easy to get the following recurrence relation,

$$
\begin{aligned}
R_{T, v}^{\sigma_{\Lambda}} & =\frac{Z_{T, v}^{\sigma_{\Lambda},+}(\zeta)}{Z_{T, v}^{\sigma_{\Lambda},-}(\zeta)}=\frac{\lambda^{1+s_{1}} \beta^{s_{1}} \prod_{i=1}^{k}\left(\beta Z_{T_{i}, v_{i}}^{\sigma_{\Lambda}^{i},+}(\zeta)+Z_{T_{i}, v_{i}}^{\sigma_{\Lambda}^{i},-}(\zeta)\right)}{\lambda^{s_{1}} \gamma^{s_{2}} \prod_{i=1}^{k}\left(Z_{T_{i}, v_{i}}^{\sigma_{\Lambda}^{i},+}(\zeta)+\gamma Z_{T_{i}, v_{i}}^{\sigma_{\Lambda}^{i},-}(\zeta)\right)} \\
& =\frac{\lambda \beta^{s_{1}}}{\gamma^{s_{2}}} \prod_{i=1}^{k}\left(\frac{\beta R_{T_{i}, v_{i}}^{\sigma_{\Lambda}^{i}}+1}{R_{T_{i}, v_{i}}^{\sigma_{i}^{i}}+\gamma}\right) .
\end{aligned}
$$

Definition 2.1 (Recursion function) Let $\mathbf{s}=\left(s_{1}, s_{2}, k\right) \in \mathbb{N}^{3}$ (including 0). A recursion function $F_{\mathrm{s}}$ for 2 -spin systems is defined to be

$$
F_{\mathbf{S}}(\zeta, \mathbf{x}):=\lambda \beta^{s_{1}} \gamma^{-s_{2}} \prod_{i=1}^{k}\left(\frac{\beta x_{i}+1}{x_{i}+\gamma}\right),
$$

where $\zeta=(\beta, \gamma, \lambda) \in \mathbb{C} \times(\mathbb{C} \backslash\{0\}) \times \mathbb{C}$ and $\mathbf{x}=\left(x_{1}, \ldots, x_{k}\right) \in(\mathbb{C} \backslash\{-\gamma\})^{k}$. We define $F_{\zeta, \mathbf{s}}(\mathbf{x}):=F_{\mathbf{s}}(\zeta, \mathbf{x})$ for fixed $\zeta$ with $\gamma \neq 0$, and $F_{\mathbf{x}, \mathbf{s}}(\zeta):=F_{\mathbf{s}}(\zeta, \mathbf{x})$ for fixed $\mathbf{x}$.

Remark Every recursion function is analytic on its domain.

For a general graph $G$, Weitz reduced computing $R_{G, v}^{\sigma_{\Lambda}}$ to that in a tree $T$, called the self-avoiding walk (SAW) tree, and Weitz's theorem [47] states that $R_{G, v}^{\sigma_{\Lambda}}=R_{T, v}^{\sigma_{\Lambda}}$. (Please see $[20,35,47]$ or the appendix for more details.) We want to generalize Weitz's theorem to complex parameters $\zeta \in \mathbb{C}^{3}$. First, we need to make sure that $R_{G, v}^{\sigma_{\Lambda}}$ and $p_{v}^{\sigma_{\Lambda}}$ are welldefined for any vertex $v \notin \Lambda$. This requires that $Z_{G}^{\sigma_{\Lambda}}(\zeta) \neq 0$ for any graph $G$ and any configuration $\sigma_{\Lambda}$. Now, $p_{v}^{\sigma_{\Lambda}}$ no longer has a probabilistic meaning. It is just a ratio of two 
complex numbers. However, one can easily observe that for some special parameters, there are trivial configurations such that $Z_{G, v}^{\sigma_{\Lambda}}(\zeta)=0$. We will rule these cases out as they are infeasible.

Definition 2.2 [Feasible configuration] Let $\zeta \in \mathbb{C}^{3}$. Given a graph $G=(V, E)$ of the 2-spin system specified by $\zeta$, a configuration $\sigma_{\Lambda}$ on some vertices $\Lambda \subseteq V$ is feasible if

- $\sigma_{\Lambda}$ does not assign any vertex in $G$ to spin + when $\lambda=0$, and

- $\sigma_{\Lambda}$ does not assign any two adjacent vertices in $G$ both to spin + when $\beta=0$.

Remark Let $\sigma_{\Lambda}$ be a feasible configuration. If we further pin one vertex $v \notin \Lambda$ to spin - , and get the configuration $\sigma_{\Lambda^{\prime}}$ on $\Lambda^{\prime}=\Lambda \cup\{v\}$, then $\sigma_{\Lambda^{\prime}}$ is still a feasible configuration. Thus, given $\zeta \in \mathbb{C}^{3}$, if $Z_{G}^{\sigma_{\Lambda}}(\zeta) \neq 0$ for any graph $G$ and any arbitrary feasible configuration $\sigma_{\Lambda}$ on $G$, then both $p_{v}^{\sigma_{\Lambda}}$ and $R_{G, v}^{\sigma_{\Lambda}}$ are well-defined.

Given $R_{G, v}^{\sigma_{\Lambda}}$ is well-defined for some $\zeta \in \mathbb{C}^{3}$, we can still compute it by recursion via the SAW tree. We first consider the case that $\lambda \neq 0$. Let $\sigma_{\Lambda}$ be a feasible configuration. It is easy to verify that the corresponding configuration on the SAW tree is also feasible and Weitz's theorem still holds. For the case that $\lambda=0$, it is obvious that $R_{G, v}^{\sigma_{\Lambda}} \equiv 0$ for any graph $G$, any free vertex $v$ and any feasible configuration $\sigma_{\Lambda}$. This is equal to the value of recursion functions $F_{\mathbf{S}}(\boldsymbol{\zeta}, \mathbf{x})$ at $\lambda=0$. We agree that $R_{G, v}^{\sigma_{\Lambda}}$ can be computed by recursion functions when $\lambda=0$, although Weitz's theorem does not hold for this case. For the case that $\beta=0$, we have $R_{G, v}^{\sigma_{\Lambda}}=0$ if one of the children of $v$ is pinned to + . Then, we may view $v$ as it is pinned to - . Thus, for $\beta=0$, we only consider recursion functions $F_{\mathrm{s}}$ where $s_{1}=0$.

\subsection{Correlation Decay}

The SAW tree may be exponentially large in size of $G$. In order to get a polynomial time approximation algorithm, we may run the tree recursion at logarithmic depth and hence in polynomial time, and plug in some arbitrary values at the truncated boundary. We have the following notion of strong spatial mixing (SSM) to bound the error caused by arbitrary guesses. It was originally introduced for non-negative parameters. Here, we extend it to complex parameters.

Definition 2.3 (Strong spatial mixing) A 2-spin system specified by $\zeta \in \mathbb{C}^{3}$ on a family $\mathcal{G}$ of graphs is said to exhibit strong spatial mixing if for any graph $G=(V, E) \in \mathcal{G}$, any $v \in V$, and any feasible configurations $\sigma_{\Lambda_{1}} \in\{0,1\}^{\Lambda_{1}}$ and $\tau_{\Lambda_{2}} \in\{0,1\}^{\Lambda_{2}}$ where $v \notin \Lambda_{1} \cup \Lambda_{2}$, we have

1. $Z_{G}^{\sigma_{\Lambda_{1}}}(\zeta) \neq 0$ and $Z_{G}^{\tau_{\Lambda_{2}}}(\zeta) \neq 0$, and

2. $\left|p_{v}^{\sigma_{\Lambda_{1}}}-p_{v}^{\tau_{\Lambda_{2}}}\right| \leq \exp (-\Omega(\operatorname{dist}(v, S)))$.

Here, $S \subseteq \Lambda_{1} \cup \Lambda_{2}$ is the subset on which $\sigma_{\Lambda_{1}}$ and $\tau_{\Lambda_{2}}$ differ (if a vertex $v$ is free in one configuration but pinned in the other, we say that these two configurations differ at $v$ ), and $\operatorname{dist}_{G}(v, S)$ is the shortest distance from $v$ to any vertex in $S$.

Remark When $\zeta \in \mathbb{R}_{+}^{3}$, condition 1 is always satisfied. Condition 2 is a stronger form of SSM of real parameters (see Definition 5 of [26]). For real values, by monotonicity one need to consider only the case that $\Lambda_{1}=\Lambda_{2}$ (the two configurations are on the same set of vertices). Here, we need to consider the case that $\Lambda_{1} \neq \Lambda_{2}$. This is necessary to extend Weitz's algorithm to complex parameters (see the proof of Lemma 3.4). 


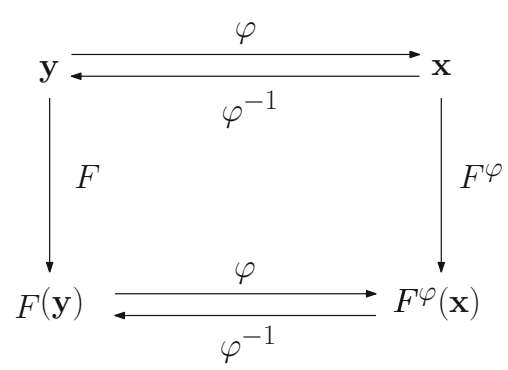

Fig. 2 Commutative diagram between $F$ and $F^{\varphi}$

In statistical physics, SSM implies correlation decay. If SSM holds, then the correlations between spins decay exponentially with the distance between them. Thus, the error caused by arbitrary boundary guesses at logarithmic depth of the SAW tree is polynomially small. Then, Weitz's algorithm gives an FPTAS. A main technique that has been widely used to establish SSM is the potential method [20,25,26,36,41]. Instead of bounding the rate of decay of recursion functions directly, we use a potential function $\varphi(x)$ to map the original recursion to a new domain (see Fig. 2 for the commutative diagram).

Let $F_{\mathbf{S}}(\boldsymbol{\zeta}, \mathbf{y})$ be a recursion function $\left(\mathbf{s}=\left(s_{1}, s_{2}, k\right) \in \mathbb{N}^{3}\right)$. We use $F_{\mathbf{S}}^{\varphi}(\boldsymbol{\zeta}, \mathbf{x})$ to denote the composition $\varphi\left(F_{\mathbf{S}}\left(\zeta, \varphi^{-1}(\mathbf{x})\right)\right)$ where $\mathbf{y}=\varphi^{-1}(\mathbf{x})$ denotes the vector $\left(\varphi^{-1}\left(x_{1}\right), \ldots, \varphi^{-1}\left(x_{k}\right)\right)$. Correspondingly, we define $F_{\zeta, \mathbf{s}}^{\varphi}(\mathbf{x})$ for fixed $\zeta$, and $F_{\mathbf{x}, \mathbf{s}}^{\varphi}(\boldsymbol{\zeta})$ for fixed $\mathbf{x}$. We will specify the domain on which $F_{\mathbf{s}}^{\varphi}$ is well-defined per each $\varphi$ that will be used.

\subsection{Real Contraction}

For positive $\zeta$, a sufficient condition for the bounded 2-spin system of $\zeta$ exhibiting SSM is that there exists a "good" potential function $\varphi$ such that $F_{\zeta, \mathrm{s}}^{\varphi}$ satisfies the following contraction property.

Definition 2.4 (Real contraction) Fix $\Delta \in \mathbb{N}$. We say that $\zeta \in \mathbb{R}^{3}$ satisfies real contraction for $\Delta$ if there is a real compact interval $J \subseteq \mathbb{R}$ where $\lambda \in J,-\gamma \notin J$ and $-1 \notin J$, and a real analytic function $\varphi: J \rightarrow I$ where $\varphi^{\prime}(x) \neq 0$ for all $x \in J$, such that

1. $F_{\boldsymbol{\zeta}, \mathbf{s}}\left(J^{k}\right) \subseteq J$ for every $\mathbf{s}$ with $\|\mathbf{s}\|_{1} \leq \Delta-1$ and $-1 \notin F_{\zeta, \mathbf{s}}\left(J^{k}\right)$ for every $\mathbf{s}$ with $\|\mathbf{s}\|_{1}=\Delta$

2. there exists $\eta>0$ s.t. $\left\|\nabla F_{\zeta, \mathbf{s}}^{\varphi}(\mathbf{x})\right\|_{1} \leq 1-\eta$ for every $\mathbf{s}$ with $\|\mathbf{s}\|_{1} \leq \Delta-1$ and all $\mathbf{x} \in I^{k}$.

We say $\varphi$ defined on $J$ is a good potential function for $\zeta$. We say a set $S \subseteq \mathbb{R}^{3}$ is a real contraction set if for every $\zeta \in S, \zeta$ satisfies real contraction for $\Delta$.

Remark Since $\varphi$ is analytic and $\varphi^{\prime}(x) \neq 0$ for all $x \in J$, the function $\varphi$ is invertible and the inverse $\varphi^{-1}: I \rightarrow J$ is also analytic by the inverse function theorem (Theorem 4.2). Also for every $\mathbf{s}$ with $\|\mathbf{s}\|_{1} \leq \Delta-1$, since $F_{\zeta, \mathbf{s}}\left(J^{k}\right) \subseteq J$ and $-\gamma \notin J$, the function $F_{\zeta, \mathbf{s}}$ is analytic on $J^{k}$. Thus, $F_{\zeta, \mathrm{s}}^{\varphi}$ is well-defined and analytic on $I^{k}$, and then $\nabla F_{\zeta, \mathrm{s}}^{\varphi}$ is well-defined on $I^{k}$. Note that $I$ is also a real compact interval since $J$ is a real compact interval and $\varphi$ is a real analytic function.

Since $-1 \notin J, F_{\zeta, \mathrm{s}}\left(J^{k}\right) \subseteq J$ implies that $-1 \notin F_{\zeta, \mathbf{s}}\left(J^{k}\right)$. Thus, real contraction implies that $-1 \notin F_{\zeta, \mathbf{s}}\left(J^{k}\right)$ for all $\|\mathbf{s}\|_{1} \leq \Delta$. The reason why we require $F_{\zeta, \mathbf{s}}\left(J^{k}\right) \subseteq J$ for $\|\mathbf{s}\|_{1} \leq$ $\Delta-1$, but only require $-1 \notin F_{\zeta, \mathbf{s}}\left(J^{k}\right)$ for $\|\mathbf{s}\|_{1}=\Delta$ is that in a tree of degree at most $\Delta$, 
only the root node may have $\Delta$ many children, while other nodes have at most $\Delta-1$ many children.

Lemma 2.5 If $\zeta \in \mathbb{R}_{+}^{3}$ satisfies real contraction for $\Delta$, then the $\Delta$-bounded 2-spin system of $\zeta$ exhibits SSM. Thus there is an FPTAS for computing the partition function $Z_{G}(\zeta)$.

Proof The proof directly follows from the argument of the potential method, see [20,26]. The FPTAS follows from Weitz's algorithm.

Now, we give the sets of non-negative parameters that satisfy real contraction.

Definition 2.6 (Uniqueness condition [26]) Let $\zeta \in \mathbb{R}^{3}$ be antiferromagnetic $(\beta \gamma<1)$ with $\beta \geq 0, \gamma>0$ and $\lambda \geq 0$, and $f_{d}(x)=\lambda\left(\frac{\beta x+1}{x+\gamma}\right)^{d}$. We say $\zeta$ is up-to- $\Delta$ unique, if $\lambda=0$ or $\lambda>0$ and there exists a constant $0<c<1$ such that for every integer $1 \leq d \leq \Delta-1$,

$$
\left|f_{d}^{\prime}\left(\hat{x}_{d}\right)\right|=\frac{d(1-\beta \gamma) \hat{x}_{d}}{\left(\beta \hat{x}_{d}+1\right)\left(\hat{x}_{d}+\gamma\right)} \leq c,
$$

where $\hat{x}_{d}$ is the unique positive fixed point of the function $f_{d}(x)$.

Let $\mathcal{S}_{i}^{\Delta}(i \in[4])$ be the correlation decay sets defined in Definition 1.2. The set $\mathcal{S}_{1}^{\Delta}$ was given in [48] and $\mathcal{S}_{2}^{\Delta}$ was given in [26]. Directly following their proofs, it is easy to verify that both sets are real contraction sets. The sets $\mathcal{S}_{3}^{\Delta}$ and $\mathcal{S}_{4}^{\Delta}$ are obtained in this paper, and we show that they are also real contraction sets.

Lemma 2.7 Fix $\Delta \geq 3$. For every $\zeta \in \mathcal{S}_{i}^{\Delta}(i \in[4])$, it satisfies real contraction for $\Delta$.

Proof We only give a proof for sets $\mathcal{S}_{3}^{\Delta}$ and $\mathcal{S}_{4}^{\Delta}$. For a proof of sets $\mathcal{S}_{1}^{\Delta}$ and $\mathcal{S}_{2}^{\Delta}$, please refer to $[26,48]$ or the appendix.

We first consider a trivial case that $\lambda=0$, in which $F_{\zeta, \mathbf{s}}(\mathbf{x}) \equiv 0$. We pick $J=[0,1]$ and the potential function $\varphi(x)=x$. Clearly, $\varphi$ is analytic on $J$ and $\varphi^{\prime}(x)=1 \neq 0$ for all $x \in J$. Also, we know $\lambda=0 \in J,-\gamma \notin J$ and $-1 \notin J$. Moreover, for every $\mathbf{s} \in \mathbb{N}^{3}$ and all $\mathbf{x} \in J^{k}$, we have $F_{\zeta, \mathbf{s}}^{\varphi}(\mathbf{x})=F_{\zeta, \mathbf{s}}(\mathbf{x}) \equiv 0 \in J$ and $\left\|\nabla F_{\zeta, \mathbf{s}}^{\varphi}(\mathbf{x})\right\|_{1} \equiv 0$. Thus, the function $\varphi$ defined on $J$ is a good potential function for $\zeta$.

Now, we consider $\lambda \neq 0$.

Since $\beta \gamma>\frac{\Delta}{\Delta-2}>1$, we have $1 / \gamma<\beta$. We pick the interval $J=\left[\lambda r^{\Delta-1}, \lambda t^{\Delta-1}\right]$ where $r=\min \{1,1 / \gamma\}$ and $t=\max \{1, \beta\}$, and the potential function $\varphi=\log (x)$. Clearly, $\varphi$ is analytic on $J$ and $\varphi^{\prime}(x) \neq 0$ for all $x \in J$. Also, we know that $\lambda \in J,-\gamma \notin J$ and $-1 \notin J$, and $-1 \notin F_{\boldsymbol{\zeta}, \mathbf{s}}\left(J^{k}\right)$ for every $\|\mathbf{s}\|_{1}=\Delta$. Since $\beta>0$ and $\gamma>0$, for all $x>0$,

$$
r \leq \min \{\beta, 1 / \gamma\} \leq \frac{\beta x+1}{x+\gamma} \leq \max \{\beta, 1 / \gamma\} \leq t .
$$

Thus, for all $\mathbf{x} \in J^{k}$,

$$
F_{\boldsymbol{\zeta}, \mathbf{s}}(\mathbf{x})=\lambda \beta^{s_{1}} \gamma^{-s_{2}} \prod_{i=1}^{k}\left(\frac{\beta x_{i}+1}{x_{i}+\gamma}\right) \in\left[\lambda r^{\|\mathbf{s}\|_{1}}, \lambda t^{\|\mathbf{s}\|_{1}}\right] \subseteq\left[\lambda r^{\Delta-1}, \lambda t^{\Delta-1}\right] .
$$

Hence, $F_{\boldsymbol{\zeta}, \mathbf{s}}\left(J^{k}\right) \subseteq J$ for every $\|\mathbf{s}\|_{1} \leq \Delta-1$. Condition 1 of real contraction is satisfied.

Let $I \stackrel{,=}{=} \varphi(J)$. Consider the gradient $\nabla F_{\zeta, \mathbf{s}}^{\varphi}(\mathbf{x})$ for every $\|\mathbf{s}\|_{1} \leq \Delta-1$ and all $\mathbf{x} \in I^{k}$. Note that $F_{\zeta, \mathbf{s}}^{\varphi}(\mathbf{x})=\log \lambda+s_{1} \log \beta-s_{2} \log \gamma+\sum_{i=1}^{k} \log \left(\frac{\beta e^{x_{i}}+1}{e^{x_{i}}+\gamma}\right)$, and $e^{x_{i}}=\varphi^{-1}\left(x_{i}\right) \in J$ and $e^{-x_{i}} \in\left[\frac{1}{\lambda t^{\Delta-1}}, \frac{1}{\lambda r^{\Delta-1}}\right]$ when $x_{i} \in I$. 
If $\zeta \in \mathcal{S}_{3}^{\Delta}$, then $(\Delta-2) \beta \gamma-\Delta<\frac{\gamma}{\lambda t^{\Delta-1}}$. Thus,

$$
\begin{aligned}
\left|\frac{\partial F_{\zeta, \mathbf{s}}^{\varphi}}{\partial x_{i}}\right| & =\frac{\beta \gamma-1}{\beta e^{x_{i}}+\gamma e^{-x_{i}}+1+\beta \gamma} \leq \frac{\beta \gamma-1}{\frac{\gamma}{\lambda t^{\Delta-1}}+1+\beta \gamma}<\frac{\beta \gamma-1}{(\Delta-2) \beta \gamma-\Delta+1+\beta \gamma} \\
& =\frac{1}{\Delta-1} .
\end{aligned}
$$

Otherwise, $\zeta \in \mathcal{S}_{4}^{\Delta}$ and then $\lambda \beta r^{\Delta-1}>(\Delta-2) \beta \gamma-\Delta$. Thus,

$$
\begin{aligned}
\left|\frac{\partial F_{\zeta, \mathrm{s}}^{\varphi}}{\partial x_{i}}\right| & =\frac{\beta \gamma-1}{\beta e^{x_{i}}+\gamma e^{-x_{i}}+1+\beta \gamma} \leq \frac{\beta \gamma-1}{\beta \lambda r^{\Delta-1}+1+\beta \gamma}<\frac{\beta \gamma-1}{(\Delta-2) \beta \gamma-\Delta+1+\beta \gamma} \\
& =\frac{1}{\Delta-1} .
\end{aligned}
$$

Thus, in both cases, there exists some $\eta>0$ such that $\left\|\nabla F_{\zeta, \mathbf{s}}^{\varphi}(\mathbf{x})\right\|_{1} \leq 1-\eta$ for every $\|\mathbf{s}\|_{1} \leq \Delta-1$ and all $\mathbf{x} \in I^{k}$. Condition 2 of real contraction is satisfied.

In order to generalize the correlation decay technique to complex parameters, we need to ensure that the partition function is zero-free. Now, let us first take a detour to Barvinok's algorithm which crucially relies on the zero-free regions of the partition function. After we carve out our new zero-free regions, we will come back to the existence of correlation decay of complex parameters.

\section{Barvinok's Algorithm}

In this section, we describe Barvinok's algorithm and introduce complex contraction.

\subsection{Zero-Freeness}

Let $I=[0, t]$ be a closed real interval. We define the $\delta$-strip of $I$ to be $\left\{z \in \mathbb{C}|| z-z_{0} \mid<\right.$ $\delta$ for some $\left.z_{0} \in I\right\}$, denoted by $I_{\delta}$. It is a complex neighborhood of $I$. Suppose a graph polynomial $P(z)=\sum_{i=0}^{n} a_{i} z^{i}$ of degree $n$ is zero-free in $I_{\delta}$. Barvinok's method [3] roughly states that for any $z \in I_{\delta}, P(z)$ can be $(1 \pm \varepsilon)$-approximated ${ }^{5}$ using coefficients $a_{0}, \ldots, a_{k}$ for some $k=O\left(e^{\Theta(1 / \delta)} \log (n / \varepsilon)\right)$, via truncating the Taylor expansion of the logarithm of the polynomial. For the partition function of 2-spin systems, these coefficients can be computed in polynomial time $[31,33]$. For the purpose of obtaining an FPTAS, we will view the partition function as a univariate polynomial $Z_{G ; \beta, \gamma}(\lambda)$ in $\lambda$ and fix $\beta$ and $\gamma$. The following result is known.

Lemma 3.1 Fix $\beta, \gamma \in \mathbb{C}$ and $\Delta \in \mathbb{N}$. Let $G$ be a graph of degree at most $\Delta$. If there is an $I=[0, t]$ such that the partition function $Z_{G ; \beta, \gamma}(\lambda) \neq 0$ for all $\lambda \in I_{\delta}$, then there is an FPTAS for computing $Z_{G ; \beta, \gamma}(\lambda)$ for all $\lambda \in I_{\delta}$.

Proof This lemma is a generalization of Lemma 4 in [19], where $\beta$ and $\gamma$ are both real. The generalization to complex valued parameters directly follows from the argument in [31].

5 For $P(z)$ evaluated at a non-real $z$, a complex value $T$ is a multiplicative $(1 \pm \varepsilon)$-approximation of $P(z)$ if $|T-P(z)| \leq \varepsilon|P(z)|$. When $\varepsilon<1$, the condition $|T-P(z)| \leq \varepsilon|P(z)|$ is equivalent to $(1-\varepsilon)|P(z)| \leq$ $|T| \leq(1+\varepsilon)|P(z)|$ and the angle between $T$ and $P(z)$ is bounded by $\arcsin \varepsilon$. 
With Lemma 3.1 in hand, the main effort is to obtain zero-free regions of the partition function. For this purpose, we will still view $Z_{G}(\zeta)$ as a multivariate polynomial in $(\beta, \gamma, \lambda)$. A main and widely used approach to obtain zero-free regions is the recursion method $[5,30$, $34,38,45]$. This method is related to the correlation decay method.

Assuming $Z_{G, v}^{-}(\zeta) \neq 0$ for some vertex $v$, then $Z_{G}(\zeta) \neq 0$ is equivalent to $R_{G, v}=$ $\frac{Z_{G, v}^{+}(\zeta)}{Z_{G, v}^{-}(\zeta)} \neq-1$. As pointed above, the ratio $R_{G, v}$ can be computed by recursion via the SAW tree in which $v$ is the root. Roughly speaking, the key idea of the recursion method is to construct a contraction region $Q \subseteq \mathbb{C}$ where $\lambda \in Q$ and $-1 \notin Q$ such that for all recursion functions $F_{\zeta, \mathbf{s}}$ with $\|\mathbf{s}\|_{1} \leq \Delta-1, F_{\zeta, \mathbf{s}}\left(Q^{k}\right) \subseteq Q$, and for all $F_{\zeta, \mathbf{s}}$ with $\|\mathbf{s}\|_{1}=\Delta$, $-1 \notin F_{\zeta, \mathrm{s}}\left(Q^{k}\right)$. This condition guarantees that with the initial value $R_{G, v_{\ell}}=\lambda$ where $v_{\ell}$ is a free leaf node in the SAW tree of which the degree is bounded by $\Delta$, the recursion will never achieve -1 . Hence, we have $Z_{G}(\zeta) \neq 0$ by induction. Again, we may use a potential function $\varphi: Q \rightarrow P$ to change the domain, and we prove $F_{\zeta, \mathrm{s}}^{\varphi}\left(P^{k}\right) \subseteq P$.

\subsection{Complex Contraction}

Now, we introduce the following complex contraction property as a generalization of real contraction. This property gives a sufficient condition for the zero-freeness of the partition function.

Definition 3.2 (Complex contraction) Fix $\Delta \in \mathbb{N}$. We say that $\zeta \in \mathbb{C}^{3}$ satisfies complex contraction for $\Delta$ if there is a closed and bounded complex region $Q \subseteq \mathbb{C}$ where $\lambda \in Q$, $-\gamma \notin Q$ and $-1 \notin Q$, and an analytic and invertible function $\varphi: Q \rightarrow P$ where the inverse $\varphi^{-1}: P \rightarrow Q$ is also analytic and $P$ is convex, such that

1. $F_{\zeta, \mathbf{s}}\left(Q^{k}\right) \subseteq Q$ for every $\mathbf{s}$ with $\|\mathbf{s}\|_{1} \leq \Delta-1$ and $-1 \notin F_{\zeta, \mathbf{s}}\left(Q^{k}\right)$ for every $\mathbf{s}$ with $\|\mathbf{s}\|_{1}=\Delta$

2. there exists $\eta>0$ s.t. $\left\|\nabla F_{\zeta, \mathbf{s}}^{\varphi}(\mathbf{x})\right\|_{1} \leq 1-\eta$ for every $\mathbf{s}$ with $\|\mathbf{s}\|_{1} \leq \Delta-1$ and all $\mathbf{x} \in P^{k}$.

We say a set $S \subseteq \mathbb{C}^{3}$ is a complex contraction set if for every $\zeta \in S, \zeta$ satisfies complex contraction for $\Delta$.

Remark Similar to the remark of Definition 2.4, the function $F_{\zeta, \mathrm{s}}^{\varphi}$ is well-defined and analytic on $P^{k}$.

Lemma 3.3 If $\zeta \in \mathbb{C}^{3}$ satisfies complex contraction for $\Delta$, then $Z_{G}^{\sigma_{\Lambda}}(\zeta) \neq 0$ for any graph $G$ of degree at most $\Delta$ and any feasible configuration $\sigma_{\Lambda}$.

Proof If $\lambda=0$, then we have $Z_{G}^{\sigma_{\Lambda}}(\zeta)=\gamma^{|E|} \neq 0$ for any graph $G=(V, E)$ and any feasible configuration $\sigma_{\Lambda}$. Hence we assume that $\lambda \neq 0$ in the rest of the proof.

By Weitz's SAW tree construction (Theorem A.1), we only need to prove this result for trees of degree at most $\Delta$. Let $t=t\left(T, \sigma_{\Lambda}\right)$ be the number of free vertices of a tree $T=(V, E)$ with a configuration $\sigma_{\Lambda}$, i.e., $t=|V|-|\Lambda|$. We prove this lemma by induction on $t$. For the base case $t\left(T, \sigma_{\Lambda}\right)=0$, we know all vertices of $T$ are pinned by $\sigma_{\Lambda}$. Since $\sigma_{\Lambda}$ is feasible, we have $Z_{T}^{\sigma_{\Lambda}}(\zeta) \neq 0$.

Now suppose that for some nonnegative integer $n$, it holds that $Z_{T^{\prime}}^{\tau_{\Lambda^{\prime}}}(\zeta) \neq 0$ for any tree $T^{\prime}$ of degree at most $\Delta$ and any feasible configuration $\tau_{\Lambda^{\prime}}$ where $t\left(T^{\prime}, \tau_{\Lambda^{\prime}}\right) \leq n$. We consider an arbitrary tree $T$ of degree at most $\Delta$ and a feasible configuration $\sigma_{\Lambda}$ where $t\left(T, \sigma_{\Lambda}\right)=n+1$. We show that $Z_{T}^{\sigma_{\Lambda}}(\zeta) \neq 0$. We pick a free vertex $v$ in $T$. By the induction hypothesis, 
we have $Z_{T, v}^{\sigma_{\Lambda},-}(\zeta) \neq 0$ since we further pinned one vertex of $T$ to spin - . Thus, the ratio $R_{T, v}^{\sigma_{\Lambda}}=\frac{Z_{T, v}^{\sigma_{\Lambda},+}(\zeta)}{Z_{T, v}^{\sigma_{\Lambda},-}(\zeta)}$ is well-defined and it can be computed by recursion (we view $v$ as the root of $T$ ). There exists an $\mathbf{s}$ with $\|\mathbf{s}\|_{1} \leq \Delta$ such that

$$
R_{T, v}^{\sigma_{\Lambda}}=F_{\zeta, \mathbf{s}}\left(R_{T_{1}, v_{1}}^{\sigma_{\Lambda}}, \ldots, R_{T_{k}, v_{k}}^{\sigma_{\Lambda}}\right)
$$

where $v_{1}, \ldots, v_{k}$ are free vertices of the children of $v$ and $T_{1}, \ldots, T_{k}$ are the corresponding subtrees rooted at them. Note that in $T$, only $v$ may have $\Delta$ many children, while other nodes have at most $\Delta-1$ many children. Therefore, for any node $v^{\prime} \neq v$ and the subtree rooted at $v^{\prime}$, the ratio $R_{T^{\prime}, v^{\prime}}^{\sigma_{\Lambda}}$ can be computed by some recursion function $F_{\boldsymbol{\zeta}, \mathbf{s}_{v^{\prime}}}$ with $\left\|\mathbf{s}_{v^{\prime}}\right\|_{1} \leq \Delta-1$. Clearly, for any free vertex $v_{\ell}$ at the leaf of $T$, we have $R_{T_{\ell}, v_{\ell}}^{\sigma_{\Lambda}}=\lambda \in Q$, where $T_{\ell}$ is a tree of only one vertex $v_{\ell}$. By complex contraction, we have $F_{\zeta, \mathbf{s}}\left(Q^{k}\right) \subseteq Q$ for every $\mathbf{s}$ with $\|\mathbf{s}\|_{1} \leq \Delta-1$. By iteration on each subtree $T_{i}$, we have $R_{T_{i}, v_{i}}^{\sigma_{\Lambda}} \in Q$ for every $i \in[k]$. Also by complex contraction, we have $-1 \notin F_{\zeta, \mathbf{s}}\left(Q^{k}\right)$ for every $\mathbf{s}$ with $\|\mathbf{s}\|_{1} \leq \Delta$. Thus, we have $R_{T, v}^{\sigma_{\Lambda}} \neq-1$. This implies that $Z_{T}^{\sigma_{\Lambda}}(\zeta) \neq 0$.

Remark The above proof only uses condition 1 of complex contraction. However, condition 2 combining with the zero-freeness result of Lemma 3.3 gives a sufficient condition for bounded 2 -spin systems of complex parameters exhibiting correlation decay. This is a generalization of Lemma 2.5.

Lemma 3.4 If $\zeta \in \mathbb{C}^{3}$ satisfies complex contraction for $\Delta$, then the $\Delta$-bounded 2-spin system of $\zeta$ exhibits SSM. Thus, there is an FPTAS for computing $Z_{G}(\zeta)$ via Weitz's algorithm.

Proof By Lemma 3.3, we know condition 1 of SSM (Definition 2.3) is satisfied. We only need to show that condition 2 is satisfied. If $\lambda=0$, then we have $p_{v}^{\sigma_{\Lambda}} \equiv 0$ for any feasible configuration $\sigma_{\Lambda}$ and SSM holds trivially. Thus, we assume that $\lambda \neq 0$. By Weitz's SAW tree construction, we only need to show that the 2-spin system on trees of degree at most $\Delta$ exhibits SSM.

Let $\varphi: P \rightarrow Q$ be a good potential function for $\zeta$. Let $T=(V, E)$ be a tree of degree at most $\Delta$ and $v$ be the root of $T$. Consider two feasible configurations $\sigma_{\Lambda_{1}}$ and $\tau_{\Lambda_{2}}$ on $\Lambda_{1} \subseteq V$ and $\Lambda_{2} \subseteq V$ respectively where $v \notin \Lambda_{1} \cup \Lambda_{2}$. We want to show that $\left|p_{v}^{\sigma_{\Lambda_{1}}}-p_{v}^{\tau_{\Lambda_{2}}}\right| \leq \exp \left(-\Omega\left(\operatorname{dist}_{T}(v, S)\right)\right)$, where $S \subseteq \Lambda_{1} \cup \Lambda_{2}$ is the subset on which $\sigma_{\Lambda_{1}}$ and $\tau_{\Lambda_{2}}$ differ. Note that all vertices in $T$ except the root $v$ have at most $\Delta-1$ many children. We first consider the case that $v$ has at most $\Delta-1$ many children. Let $t=\operatorname{dist}_{T}(v, S)$. We will show

$$
\left|\varphi\left(R_{T, v}^{\sigma_{\Lambda_{1}}}\right)-\varphi\left(R_{T, v}^{\tau_{\Lambda_{2}}}\right)\right| \leq C(1-\eta)^{t-1}
$$

for some constants $C, \eta>0$ by induction on $t$. Here, $C$ only depends on the convex domain $P$, while $\eta$ depends on the good potential function $\phi$.

For the base case $t=1$, since $\zeta$ satisfies complex contraction, we have $R_{T, v}^{\sigma_{\Lambda_{1}}}, R_{T, v}^{\tau_{\Lambda_{2}}} \in Q$ and hence $\varphi\left(R_{T, v}^{\sigma_{\Lambda_{1}}}\right), \varphi\left(R_{T, v}^{\tau_{\Lambda_{2}}}\right) \in P$. Let $C=\sup _{z_{1}, z_{2} \in P}\left|z_{1}-z_{2}\right|$. Since $P$ is a closed and bounded region, $C=\max _{z_{1}, z_{2} \in P}\left|z_{1}-z_{2}\right|<+\infty$. Clearly, $\left|\varphi\left(R_{T, v}^{\sigma_{\Lambda_{1}}}\right)-\varphi\left(R_{T, v}^{\tau_{\Lambda_{2}}}\right)\right| \leq C$.

Since $\zeta$ satisfies complex contraction, let $\eta$ be the constant such that $\left\|\nabla F_{\zeta, \mathbf{s}}^{\varphi}(\mathbf{x})\right\|_{1} \leq 1-\eta$ for every $\mathbf{s}$ with $\|\mathbf{s}\|_{1} \leq \Delta-1$ and all $\mathbf{x} \in P^{k}$. Suppose that $\left|\varphi\left(R_{T, v}^{\sigma_{\Lambda_{1}}}\right)-\varphi\left(R_{T, v}^{\tau_{\Lambda_{2}}}\right)\right| \leq$ $C(1-\eta)^{t-1}$ for $t \leq n$ where $n$ is a positive integer. We consider $t=n+1$. Since $t>1$, the 
configurations of all children of $v$ are the same in both $\sigma_{\Lambda_{1}}$ and $\tau_{\Lambda_{2}}$. Suppose that $v$ has $d$ children, and in both configurations $\sigma_{\Lambda_{1}}$ and $\tau_{\Lambda_{2}}, s_{1}$ of them are pinned to,$+ s_{2}$ are pinned to - , and $k$ are free. We denote these $k$ free vertices by $v_{i}(i \in[k])$. Let $T_{i}$ be the corresponding subtree rooted at $v_{i}$, and $\sigma_{\Lambda_{1}}^{i}$ and $\tau_{\Lambda_{2}}^{i}$ denote the configurations $\sigma_{\Lambda_{1}}$ and $\tau_{\Lambda_{2}}$ restricted on the subtree $T_{i}$ respectively. Let $x_{i}=\varphi\left(R_{T_{i}, v_{i}}^{\sigma_{\Lambda_{1}}^{i}}\right)$ and $y_{i}=\varphi\left(R_{T_{i}, v_{i}}^{\tau_{\Lambda_{2}}^{i}}\right)$. Since $\zeta$ satisfies complex contraction, same as we showed in the proof of Lemma 3.3, we have $R_{T_{i}, v_{i}}^{\sigma_{\Lambda_{1}}^{i}}, R_{T_{i}, v_{i}}^{\tau_{\Lambda_{2}}^{i}} \in Q$ and hence $x_{i}, y_{i} \in P$. Let $S_{i}=S \cap T_{i}$. Clearly, we have $\operatorname{dist}_{T_{i}}\left(v_{i}, S_{i}\right) \geq \operatorname{dist}_{T}(v, S)-1=t-1=$ $n$. By induction hypothesis, we have $\left|x_{i}-y_{i}\right| \leq C(1-\eta)^{\operatorname{dist}_{T_{i}}\left(v_{i}, S_{i}\right)-1} \leq C(1-\eta)^{n-1}$. Then, we have

$$
\begin{aligned}
\left|\varphi\left(R_{T, v}^{\sigma_{\Lambda_{1}}}\right)-\varphi\left(R_{T, v}^{\tau_{\Lambda_{2}}}\right)\right| & =\left|\varphi\left(F_{\zeta, \mathbf{s}}\left(R_{T_{1}, v_{1}}^{\sigma_{\Lambda_{1}}^{1}}, \ldots, R_{T_{k}, v_{k}}^{\sigma_{\Lambda_{1}}^{k}}\right)\right)-\varphi\left(F_{\zeta, \mathbf{s}}\left(R_{T_{1}, v_{1}}^{\tau_{\Lambda_{2}}^{1}}, \ldots, R_{T_{k}, v_{k}}^{\tau_{\Lambda_{2}}^{k}}\right)\right)\right| \\
& =\left|F_{\zeta, \mathbf{s}}^{\varphi}\left(x_{1}, \ldots, x_{k}\right)-F_{\zeta, \mathbf{s}}^{\varphi}\left(y_{1}, \ldots, y_{k}\right)\right| \\
& \leq \sup _{\mathbf{z} \in P^{k}}\left\|\nabla F_{\zeta, \mathbf{s}}^{\varphi}(\mathbf{z})\right\|_{1} \cdot\|\mathbf{x}-\mathbf{y}\|_{\infty} \\
& \leq(1-\eta) \cdot C(1-\eta)^{n-1} \\
& =C(1-\eta)^{t-1},
\end{aligned}
$$

where the first inequality is due to the fundamental theorem of calculus and the fact that $P$ is convex.

Then, we bound $\left|R_{T, v}^{\sigma_{\Lambda_{1}}}-R_{T, v}^{\tau_{\Lambda_{2}}}\right|$. Define functions $G_{\zeta, \mathrm{s}}(\mathbf{x}):=F_{\zeta, \mathrm{s}}\left(\varphi^{-1}(\mathbf{x})\right)$. Since $F_{\zeta, \mathrm{s}}$ is analytic on $Q^{k}$, we have $G_{\zeta, \mathbf{s}}$ is well-defined and analytic on $P^{k}$ for all $\|\mathbf{s}\|_{1} \leq \Delta$. For every $\mathbf{s}$ with $\|\mathbf{s}\|_{1} \leq \Delta$, let $Q_{\mathbf{s}}=G_{\zeta, \mathbf{s}}\left(P^{k}\right)=F_{\boldsymbol{\zeta}, \mathbf{s}}\left(Q^{k}\right)$ and $M_{\mathbf{s}}=\sup _{\mathbf{x} \in P^{k}}\left\|\nabla G_{\zeta, \mathbf{s}}(\mathbf{x})\right\|_{1}$. Since $P^{k}$ is compact (closed and bounded), we have $Q_{\mathbf{s}}$ is compact and $M_{\mathbf{s}}<+\infty$. Finally, let

$$
Q^{\prime}=\bigcup_{\|\mathbf{s}\|_{1} \leq \Delta} Q_{\mathbf{s}}, \quad C^{\prime}=\sup _{z_{1}, z_{2} \in Q^{\prime}}\left|z_{1}-z_{2}\right|, \quad \text { and } \quad M=\max _{\|\mathbf{s}\|_{1} \leq \Delta} M_{\mathbf{s}} .
$$

Since there is only a finite number of $\mathbf{s}$ such that $\|\mathbf{s}\|_{1} \leq \Delta$, we have $M<+\infty$ and $Q^{\prime}$ is compact, and hence $C^{\prime}<+\infty$. Let $N=\max \left\{C^{\prime}, C M\right\}$. We show that $\left|R_{T, v}^{\sigma_{\Lambda_{1}}}-R_{T, v}^{\tau_{\Lambda_{2}}}\right| \leq$ $N(1-\eta)^{t-2}$.

If $t=1$, then there exist $\mathbf{s}_{1}$ and $\mathbf{s}_{2}$ where $\left\|\mathbf{s}_{1}\right\|_{1},\left\|\mathbf{s}_{2}\right\|_{1} \leq \Delta$ such that $R_{T, v}^{\sigma_{\Lambda_{1}}} \in F_{\zeta, \mathbf{s}_{1}}\left(Q^{k_{1}}\right)$ and $R_{T, v}^{\tau_{\Lambda_{2}}} \in F_{\boldsymbol{\zeta}, \mathbf{s}_{2}}\left(Q^{k_{2}}\right)$. Then, we have $R_{T, v}^{\sigma_{\Lambda_{1}}}, R_{T, v}^{\tau_{\Lambda_{2}}} \in Q^{\prime}$, and hence

$$
\left|R_{T, v}^{\sigma_{\Lambda_{1}}}-R_{T, v}^{\tau_{\Lambda_{2}}}\right| \leq C^{\prime} \leq \frac{N}{1-\eta}
$$

Otherwise $t>1$. The configurations of all children of $v$ are the same in both $\sigma_{\Lambda_{1}}$ and $\tau_{\Lambda_{2}}$. Again, let $x_{i}=\varphi\left(R_{T_{i}, v_{i}}^{\sigma_{\Lambda_{1}}^{i}}\right)$ and $y_{i}=\varphi\left(R_{T_{i}, v_{i}}^{\tau_{\Lambda_{2}}^{i}}\right)$, where $v_{i}, T_{i}, \sigma_{\Lambda_{1}}^{i}, \tau_{\Lambda_{2}}^{i}$ and $S_{i}(i \in[k])$ are all defined the same as in the above induction proof. Since in the subtree $T_{i}$, the root $v_{i}$ has at most $\Delta-1$ many children, we have

$$
\left|x_{i}-y_{i}\right| \leq C(1-\eta)^{\operatorname{dist}_{T_{i}}\left(v_{i}, S_{i}\right)-1} \leq C(1-\eta)^{t-2} .
$$


Then, similarly as we did in the above induction proof, we have

$$
\begin{aligned}
\left|R_{T, v}^{\sigma_{\Lambda_{1}}}-R_{T, v}^{\tau_{\Lambda_{2}}}\right| & =\left|F_{\zeta, \mathbf{s}}\left(R_{T_{1}, v_{1}}^{\sigma_{\Lambda_{1}}^{1}}, \ldots, R_{T_{k}, v_{k}}^{\sigma_{\Lambda_{1}}^{k}}\right)-F_{\boldsymbol{\zeta}, \mathbf{s}}\left(R_{T_{1}, v_{1}}^{\tau_{\Lambda_{2}}^{1}}, \ldots, R_{T_{k}, v_{k}}^{\tau_{\Lambda_{2}}^{k}}\right)\right| \\
& =\left|G_{\zeta, \mathbf{s}}\left(x_{1}, \ldots, x_{k}\right)-G_{\boldsymbol{\zeta}, \mathbf{s}}\left(y_{1}, \ldots, y_{k}\right)\right| \\
& \leq \sup _{\mathbf{x} \in P^{k}}\left\|\nabla G_{\zeta, \mathbf{s}}(\mathbf{x})\right\|_{1} \cdot\|\mathbf{x}-\mathbf{y}\|_{\infty} \\
& \leq M \cdot C(1-\eta)^{t-2} \\
& \leq N(1-\eta)^{t-2} .
\end{aligned}
$$

Finally, we bound $\left|p_{v}^{\sigma_{\Lambda_{1}}}-p_{v}^{\tau_{\Lambda_{2}}}\right|$ from $\left|R_{T, v}^{\sigma_{\Lambda_{1}}}-R_{T, v}^{\tau_{\Lambda_{2}}}\right|$. Let $K=\inf _{z \in Q^{\prime}}|1+z|$. By the complex contraction property, $-1 \notin Q_{\mathbf{s}}$ for every $\|\mathbf{s}\|_{1} \leq \Delta$ and thus $-1 \notin Q^{\prime}$. Also, since $Q^{\prime}$ is compact, we have $K>0$. Hence,

$$
\begin{aligned}
\left|p_{v}^{\sigma_{\Lambda_{1}}}-p_{v}^{\tau_{\Lambda_{2}}}\right| & =\left|\frac{R_{T, v}^{\sigma_{\Lambda_{1}}}}{1+R_{T, v}^{\sigma_{\Lambda_{1}}}}-\frac{R_{T, v}^{\tau_{\Lambda_{2}}}}{1+R_{T, v}^{\tau_{\Lambda_{2}}}}\right|=\frac{\left|R_{T, v}^{\sigma_{\Lambda_{1}}}-R_{T, v}^{\tau_{\Lambda_{2}}}\right|}{\left|1+R_{T, v}^{\sigma_{\Lambda_{1}}}\right| \cdot\left|1+R_{T, v}^{\tau_{\Lambda_{2}}}\right|} \\
& \leq \frac{1}{K^{2}} \cdot\left|R_{T, v}^{\sigma_{\Lambda_{1}}}-R_{T, v}^{\tau_{\Lambda_{2}}}\right| \leq \frac{N(1-\eta)^{t-2}}{K^{2}} \\
& \leq \exp \left(-\Omega\left(\operatorname{dist}_{T}(v, S)\right) .\right.
\end{aligned}
$$

\section{From Real Contraction to Complex Contraction}

In this section, we prove our main result. We first give some preliminaries in complex analysis. The main tools are the unique analytic continuation and the inverse function theorem. Here, we slightly modify the statements to fit for our settings. Please refer to [46] for the proofs.

Theorem 4.1 (Unique analytic continuation) Let $f(x)$ be a (real) analytic function defined on a compact real interval $I \subseteq \mathbb{R}$. Then there exists a complex neighborhood $\widetilde{I} \subseteq \mathbb{C}$ of $I$, and a (complex) analytic function $\widetilde{f}(x)$ defined on $\widetilde{I}$ such that $\widetilde{f}(x) \equiv f(x)$ for all $x \in I$. Moreover, if there is another (complex) analytic function $\widetilde{g}(x)$ also defined on $\widetilde{I}$ such that $\widetilde{g}(x) \equiv \widetilde{f}(x)$ for all $x \in I$ and the measure $\mathfrak{m}(I) \neq 0$ (i.e., I is not a singleton), then $\widetilde{g}(x) \equiv \widetilde{f}(x)$ for all $x \in \widetilde{I}$. We call $\widetilde{f}(x)$ the unique analytic continuation of $f(x)$ on $\widetilde{I}$.

Theorem 4.2 (Inverse function theorem) For a real analytic function $\varphi$ defined on a real interval $J \subseteq \mathbb{R}$, if $\varphi^{\prime}(x) \neq 0$ for all $x \in J$, then $\varphi$ is invertible on $J$ and the inverse $\varphi^{-1}$ is also analytic on $\varphi(J)$. For a complex analytic function $\psi$ defined on $U \subseteq \mathbb{C}$, if $\psi^{\prime}(z) \neq 0$ for some $z \in U$, then there exists a complex neighborhood $D$ of $z$ such that $\psi$ is invertible on $D$ and the inverse is also analytic.

Combining the above theorems, we have the following result.

Lemma 4.3 Let $\varphi: J \rightarrow I$ be a real analytic function, and $\varphi^{\prime}(x) \neq 0$ for all $x \in J$ where $J$ and $I$ are real compact intervals. Then, there exists an analytic continuation $\tilde{\varphi}$ on a complex neighborhood $\widetilde{J}$ of $J$ such that $\widetilde{\varphi}$ is invertible on $\widetilde{J}$ and the inverse $\widetilde{\varphi}^{-1}$ is also analytic. 
Proof If $\mathfrak{m}(J)=0$, i.e., $J=\{x\}$, then by Theorem 4.1, there exists an analytic continuation $\widetilde{\varphi}$ of $\varphi$. Since $\widetilde{\varphi}^{\prime}(x)=\varphi^{\prime}(x) \neq 0$, by Theorem 4.2, there is a neighborhood of $x$ on which $\tilde{\varphi}$ is invertible and the inverse $\widetilde{\varphi}^{-1}$ is analytic.

Otherwise, $\mathfrak{m}(J) \neq 0$. Since $\varphi(x)$ is analytic and $\varphi^{\prime}(x) \neq 0$ for all $x \in J$, the function $\varphi$ is invertible and by Theorem 4.2, the inverse $\varphi^{-1}: I \rightarrow J$ is analytic on $I$. By Theorem 4.1, there exists an analytic continuation $\widetilde{\varphi^{-1}}$ of $\varphi^{-1}$ defined on a neighborhood $\widetilde{I}_{1}$ of $I$. Similarly, there exists an analytic continuation $\widetilde{\varphi}$ of $\varphi$ defined on a neighborhood $\widetilde{J}$ of $J$. We use $\widetilde{I}$ to denote the image $\widetilde{\varphi}(\widetilde{J})$. Since $\widetilde{\varphi}$ is analytic, by the open mapping theorem $\widetilde{I}$ is an open set in the complex plane. Clearly, we have $\varphi(J)=I \subseteq \widetilde{I}$. We can pick $\widetilde{J}$ small enough while still keeping $J \subseteq \widetilde{J}$ such that the image $\widetilde{I}=\widetilde{\varphi}(\widetilde{J}) \subseteq \widetilde{I}_{1}$ and still $I \subseteq \widetilde{I}$. Thus, the composition $\widetilde{\varphi^{-1}} \circ \widetilde{\varphi}$ is a well-defined analytic function on $\widetilde{J}$. Clearly,

$$
\widetilde{\varphi^{-1}} \circ \widetilde{\varphi}(x)=\varphi^{-1} \circ \varphi(x) \equiv x \text { for all } x \in J .
$$

Since $\mathfrak{m}(J) \neq 0$, by Theorem 4.1, we have that $\widetilde{\varphi^{-1}} \circ \widetilde{\varphi}(x) \equiv x$ for all $x \in \widetilde{J}$.

Thus, $\widetilde{\varphi}$ is invertible on $\widetilde{J}$ and the inverse $\widetilde{\varphi}^{-1}=\widetilde{\varphi^{-1}}$ is analytic.

Now, we are ready to prove our main result.

Theorem 4.4 If $\zeta_{0}$ satisfies real contraction for $\Delta$, then there exists a $\delta>0$ such that for every $\zeta \in \mathbb{C}^{3}$ with $\left\|\zeta-\zeta_{0}\right\|_{\infty}<\delta$, $\zeta$ satisfies complex contraction for $\Delta$.

Proof Let $\varphi: J \rightarrow I$ be a good potential function for $\zeta_{0}$. By Definition 2.4 and Lemma 4.3 , there exists a neighborhood $\widetilde{J}$ of $J$ such that the analytic continuation $\widetilde{\varphi}: \widetilde{J} \rightarrow \widetilde{I}$ of $\varphi$ on $\widetilde{J}$ is invertible. Here $\widetilde{I}=\widetilde{\varphi}(\widetilde{J})$ is a neighborhood of $I$, and the inverse $\widetilde{\varphi}^{-1}$ is also analytic on $\widetilde{I}$. We use $\mathcal{B}_{\delta}:=\left\{\mathbf{z} \in \mathbb{C}^{3} \mid\left\|\mathbf{z}-\zeta_{0}\right\|_{\infty}<\delta\right\}$ to denote the 3-dimensional complex ball around $\zeta_{0}$ of radius $\delta$ in terms of the infinity norm. Recall that we define $I_{\varepsilon}=\left\{z \in \mathbb{C}|| z-z_{0} \mid<\varepsilon\right.$ for some $\left.z_{0} \in I\right\}$. Given a set $U \subseteq \mathbb{C}^{k}$, we use $\bar{U}$ to denote its closure.

We first show that we can pick a pair of $\left(\delta_{1}, \varepsilon_{1}\right)$ such that for every $\mathbf{s}$ with $\|\mathbf{S}\|_{1} \leq \Delta-1$, the composition

$$
F_{\mathbf{s}}^{\widetilde{\varphi}}(\boldsymbol{\zeta}, \mathbf{x})=\widetilde{\varphi}\left(F_{\mathbf{S}}\left(\zeta, \widetilde{\varphi}^{-1}(\mathbf{x})\right)\right) \text { is well-defined and analytic on } \mathcal{B}_{\delta_{1}} \times I_{\varepsilon_{1}}^{k} .
$$

Given some $\mathbf{s}$ with $\|\mathbf{s}\|_{1} \leq \Delta-1$, we consider the function $F_{\mathbf{s}}(\zeta, \mathbf{x})$. We know that it is analytic on a neighborhood of $\left\{\boldsymbol{\zeta}_{0}\right\} \times J^{k}$ and by real contraction we have $F_{\mathbf{s}}\left(\zeta_{0}, J^{k}\right) \subseteq J$. Then, we can pick some $\delta_{\mathbf{s}}$ and a neighborhood $\widetilde{J}_{\mathbf{s}}$ of $J$ that are small enough such that $F_{\mathbf{s}}(\zeta, \mathbf{x})$ is analytic on $\mathcal{B}_{\delta_{\mathbf{s}}} \times \widetilde{J}_{\mathbf{s}}^{k}$, and $F_{\mathbf{s}}\left(\mathcal{B}_{\delta_{\mathbf{s}}}, \widetilde{J}_{\mathbf{s}}^{k}\right) \subseteq \widetilde{J}$. Let

$$
\delta_{1}=\min _{\|\mathbf{s}\|_{1} \leq \Delta-1}\left\{\delta_{\mathbf{s}}\right\} \quad \text { and } \quad \widetilde{J}_{1}=\bigcap_{\|\mathbf{s}\|_{1} \leq \Delta-1} \widetilde{J}_{\mathbf{s}} .
$$

Since there is only a finite number of $\mathbf{s}$ with $\|\mathbf{s}\|_{1} \leq \Delta-1$, we have that $\delta_{1}>0$, and $\widetilde{J}_{1}$ is open and it is a neighborhood of $J$. Then, $F_{\mathbf{s}}\left(\mathcal{B}_{\delta_{1}}, \widetilde{J}_{1}\right) \subseteq \widetilde{J}$ for every $\mathbf{s}$ with $\|\mathbf{s}\|_{1} \leq \Delta-1$. Since $\widetilde{\varphi}^{-1}$ is analytic on $\widetilde{I}$ and $\widetilde{\varphi}^{-1}(I)=J$, similarly we can pick a small enough neighborhood $\widetilde{I}_{1}$ of $I$ where $\widetilde{I}_{1} \subseteq \widetilde{I}$ such that $\widetilde{\varphi}^{-1}\left(\widetilde{I}_{1}\right) \subseteq \widetilde{J}_{1}$. For every $z_{0} \in I$, we can pick an $\varepsilon_{z_{0}}$ such that the disc $B_{z_{0}, \varepsilon_{z_{0}}}:=\left\{z \in \mathbb{C}|| z-z_{0} \mid<\varepsilon_{z_{0}}\right\}$ is in $\widetilde{I}_{1}$. Recall that $I$ is a compact real interval, by the finite cover theorem, we can uniformly pick an $\varepsilon_{1}$ such that $I \subseteq I_{\varepsilon_{1}} \subseteq \widetilde{I}_{1}$. Thus, $F_{\mathbf{S}}^{\widetilde{\varphi}}(\boldsymbol{\zeta}, \mathbf{x})$ is well-defined and analytic on $\mathcal{B}_{\delta_{1}} \times I_{\varepsilon_{1}}^{k}$ for every $\mathbf{s}$ with $\|\mathbf{s}\|_{1} \leq \Delta-1$. In fact, $F_{\mathbf{S}}^{\widetilde{\varphi}}$ is a (multivariate) analytic continuation of $F_{\mathbf{s}}^{\varphi}$. Since $I$ is a compact interval, in the 
following when we pick a neighborhood $\widetilde{I}$ of $I$, without loss of generality, we may always pick $\widetilde{I}$ as an $\varepsilon$-strip $I_{\varepsilon}$ of $I$.

Then, we show that we can pick a pair of $\left(\delta_{2}, \varepsilon_{2}\right)$ where $\delta_{2}<\delta_{1}$ and $\varepsilon_{2}<\varepsilon_{1}$, a constant $M>0$ and a constant $\eta>0$ such that for every $\mathbf{s}$ with $\|\mathbf{s}\|_{1} \leq \Delta-1$,

$$
\left\|\nabla F_{\zeta, \mathbf{s}}^{\widetilde{\varphi}}(\mathbf{x})\right\|_{1} \leq 1-\eta \quad \text { and } \quad\left\|\nabla F_{\mathbf{x}, \mathbf{s}}^{\widetilde{\varphi}}(\zeta)\right\|_{1} \leq M
$$

for all $\zeta \in \overline{\mathcal{B}_{\delta_{2}}}$ and all $\mathbf{x} \in \overline{I_{\varepsilon_{2}}^{k}}$. By real contraction, there is an $\eta^{\prime}>0$ such that $\left\|\nabla F_{\boldsymbol{\zeta}_{0}, \mathbf{s}}^{\widetilde{\varphi}}(\mathbf{x})\right\|_{1} \leq 1-\eta^{\prime}$ for every $\mathbf{s}$ with $\|\mathbf{s}\|_{1} \leq \Delta-1$ and all $\mathbf{x} \in I^{k}$. Given some $\mathbf{s}$ with $\|\mathbf{s}\|_{1} \leq \Delta-1$, since $F_{\mathbf{s}}^{\widetilde{\varphi}}(\boldsymbol{\zeta}, \mathbf{x})$ is analytic on $\mathcal{B}_{\delta_{1}} \times I_{\varepsilon_{1}}^{k}$, by continuity we can pick some $\delta_{\mathbf{s}}<\delta_{1}$ and $\varepsilon_{\mathbf{s}}<\varepsilon_{1}$ such that $\left\|\nabla F_{\zeta, \mathbf{s}}^{\widetilde{\varphi}}(\mathbf{x})\right\|_{1} \leq 1-\frac{\eta^{\prime}}{2}$ for all $\zeta \in \overline{\mathcal{B}_{\delta_{\mathbf{s}}}}$ and all $\mathbf{x} \in \overline{I_{\varepsilon_{\mathbf{s}}}^{k}}$. In addition, let

$$
M_{\mathbf{s}}=\sup _{\zeta \in \overline{\mathcal{B}_{\delta_{\mathbf{s}}}}, \mathbf{x} \in \overline{I_{\varepsilon_{\mathbf{s}}}^{k}}}\left\|\nabla F_{\mathbf{x}, \mathbf{s}}^{\widetilde{\varphi}}(\zeta)\right\|_{1},
$$

and we know that $M_{\mathbf{S}}<+\infty$ since $F^{\widetilde{\varphi}}$ is analytic on $\overline{\mathcal{B}_{\delta_{\mathbf{S}}}} \times \overline{I_{\varepsilon_{\mathbf{S}}}^{k}}$ which is closed and bounded. Finally, let

$$
\eta=\frac{\eta^{\prime}}{2}, \quad \delta_{2}=\min _{\|\mathbf{s}\|_{1} \leq \Delta-1}\left\{\delta_{\mathbf{s}}\right\}, \quad \varepsilon_{2}=\min _{\|\mathbf{s}\|_{1} \leq \Delta-1}\left\{\varepsilon_{\mathbf{s}}\right\}, \quad \text { and } \quad M=\max _{\|\mathbf{s}\|_{1} \leq \Delta-1}\left\{M_{\mathbf{s}}\right\} .
$$

These choices will satisfy our requirement.

For the case that $\|\mathbf{s}\|_{1}=\Delta$, we show that we can pick a pair of $\left(\delta_{3}, \varepsilon_{3}\right)$ where $\delta_{3}<\delta_{1}$ and $\varepsilon_{3}<\varepsilon_{1}$ such that for every $\mathbf{s}$ with $\|\mathbf{s}\|_{1}=\Delta$, we have $-1 \notin F_{\mathbf{S}}\left(\overline{\mathcal{B}_{\delta_{3}}}, \widetilde{J}_{2}^{k}\right)$ where $\widetilde{J}_{2}=\widetilde{\varphi}^{-1}\left(\overline{I_{\varepsilon_{3}}}\right)$ is a closed neighborhood of $J$. Since $F_{\mathrm{s}}$ is analytic, by real contraction, $-1 \notin F_{\zeta_{0}, \mathrm{~s}}\left(J^{k}\right)$ which is closed. Again by continuity we can pick some $\left(\delta_{3}, \varepsilon_{3}\right)$ that satisfy our requirement.

Since $\zeta_{0}=\left(\beta_{0}, \gamma_{0}, \lambda_{0}\right)$ satisfies real contraction, we have $\lambda_{0} \in J,-\gamma_{0} \notin J$ and $-1 \notin J$. Recall that $J=\widetilde{\varphi}^{-1}(I)$. Again, since $\widetilde{\varphi}^{-1}$ is analytic, by continuity we can pick some $\varepsilon \leq \min \left\{\varepsilon_{2}, \varepsilon_{3}\right\}$ such that $\lambda_{0} \in \widetilde{\varphi}^{-1}\left(I_{\varepsilon}\right),-\gamma_{0} \notin \widetilde{\varphi}^{-1}\left(\overline{I_{\varepsilon}}\right)$ (note that $\widetilde{\varphi}^{-1}\left(\overline{I_{\varepsilon}}\right)$ is a closed set) and $-1 \notin \widetilde{\varphi}^{-1}\left(\overline{I_{\varepsilon}}\right)$. Moreover, we can pick some $\delta_{4}$ small enough such that the disc $B_{\lambda_{0}, \delta_{4}}:=$ $\left\{z \in \mathbb{C}|| z-\lambda_{0} \mid<\delta_{4}\right\}$ is in $\widetilde{\varphi}^{-1}\left(I_{\varepsilon}\right)$, and the disc $B_{-\gamma_{0}, \delta_{4}}:=\left\{z \in \mathbb{C}|| z-\left(-\gamma_{0}\right) \mid<\delta_{4}\right\}$ is disjoint with $\widetilde{\varphi}^{-1}\left(\overline{I_{\varepsilon}}\right)$. Let $P=\overline{I_{\varepsilon}}$ and $Q=\widetilde{\varphi}^{-1}\left(\overline{I_{\varepsilon}}\right)$. Clearly, $P$ is convex. For every $\zeta$ with $\left\|\zeta-\zeta_{0}\right\|_{\infty}<\delta$, we have $\lambda \in Q,-\gamma \notin Q$ and $-1 \notin Q$. In addition, we know that $Q$ is closed and bounded since $P$ is closed and bounded and $\tilde{\varphi}^{-1}$ is analytic on $P$. Finally, let $\delta=\min \left\{\delta_{2}, \delta_{3}, \delta_{4}, \frac{\varepsilon \eta}{M}\right\}$. We show that for every $\mathbf{s}$ with $\|\mathbf{s}\|_{1} \leq \Delta-1, F_{\mathbf{s}}^{\widetilde{\varphi}}\left(\mathcal{B}_{\delta}, P^{k}\right) \subseteq P$, which implies that $F_{\mathbf{s}}\left(\mathcal{B}_{\delta}, Q^{k}\right) \subseteq Q$.

Consider some $\mathbf{x} \in P^{k}$. By the definition of $P$, there exists an $\mathbf{x}_{0} \in I^{k}$ such that $\| \mathbf{x}-$ $\mathbf{x}_{0} \|_{\infty} \leq \varepsilon$. Also, consider some $\zeta \in \mathcal{B}_{\delta}$, and we have $\left\|\zeta-\zeta_{0}\right\|_{\infty}<\delta$. Then, for every $\mathbf{s}$ with $\|\mathbf{s}\|_{1} \leq \Delta-1$, consider $F_{\mathbf{s}}^{\widetilde{\varphi}}(\boldsymbol{\zeta}, \mathbf{x})-F_{\mathbf{s}}^{\widetilde{\varphi}}\left(\zeta_{0}, \mathbf{x}_{0}\right)$. We have

$$
\begin{aligned}
& \left|F_{\mathbf{s}}^{\widetilde{\varphi}}(\boldsymbol{\zeta}, \mathbf{x})-F_{\mathbf{s}}^{\widetilde{\varphi}}\left(\zeta_{0}, \mathbf{x}_{0}\right)\right| \\
& \quad \leq\left|F_{\mathbf{s}}^{\widetilde{\varphi}}(\boldsymbol{\zeta}, \mathbf{x})-F_{\mathbf{s}}^{\widetilde{\varphi}}\left(\boldsymbol{\zeta}_{0}, \mathbf{x}\right)\right|+\left|F_{\mathbf{s}}^{\widetilde{\varphi}}\left(\boldsymbol{\zeta}_{0}, \mathbf{x}\right)-F_{\mathbf{s}}^{\widetilde{\varphi}}\left(\boldsymbol{\zeta}_{0}, \mathbf{x}_{0}\right)\right| \\
& \quad \leq \sup _{\zeta^{\prime} \in \mathcal{B}_{\delta}}\left\|\nabla F_{\mathbf{x}, \mathbf{s}}^{\widetilde{\varphi}}\left(\zeta^{\prime}\right)\right\|_{1} \cdot\left\|\zeta-\zeta_{0}\right\|_{\infty}+\sup _{\mathbf{x}^{\prime} \in P^{k}}\left\|\nabla F_{\zeta_{0}, \mathbf{s}}^{\widetilde{\varphi}}\left(\mathbf{x}^{\prime}\right)\right\|_{1} \cdot\left\|\mathbf{x}-\mathbf{x}_{0}\right\|_{\infty} \\
& \quad \leq M \delta+(1-\eta) \cdot \varepsilon \leq \varepsilon .
\end{aligned}
$$

The second inequality above uses the fact that both $\mathcal{B}_{\delta}$ and $P^{k}$ are convex, which ensures that the line between $\zeta_{0}$ and $\zeta$ is in $\mathcal{B}_{\delta}$ and the line between $\mathbf{x}_{0}$ and $\mathbf{x}$ is in $P^{k}$. By real contraction, 
we know that $F_{\mathbf{s}}^{\widetilde{\varphi}}\left(\zeta_{0}, \mathbf{x}_{0}\right) \in I$ since $\mathbf{x}_{0} \in I^{k}$. Thus, we have $F_{\mathbf{s}}^{\widetilde{\varphi}}(\zeta, \mathbf{x}) \in P$. Thus, for every $\zeta$ with $\left\|\zeta-\zeta_{0}\right\|_{\infty}<\delta$, we have that $\lambda \in Q,-\gamma \notin Q$ and $-1 \notin Q$, and

1. $F_{\zeta, \mathbf{s}}\left(Q^{k}\right) \subseteq Q$ for every $\mathbf{s}$ with $\|\mathbf{s}\|_{1} \leq \Delta-1$ and $-1 \notin F_{\zeta, \mathbf{s}}\left(Q^{k}\right)$ for every $\mathbf{s}$ with $\|\mathbf{s}\|_{1}=\Delta$

2. there exists $\eta>0$ s.t. $\left\|\nabla F_{\zeta, \mathbf{s}}^{\varphi}(\mathbf{x})\right\|_{1} \leq 1-\eta$ for every $\mathbf{s}$ with $\|\mathbf{s}\|_{1} \leq \Delta-1$ and all $\mathbf{x} \in P^{k}$.

The function $\widetilde{\varphi}: Q \rightarrow P$ is a good potential function for $\zeta$.

Combining Lemmas 2.7, 3.3, 3.4 and Theorem 4.4, we have the following result.

Theorem 4.5 Fix $\Delta \geq 3$. For every $\zeta_{0} \in \mathcal{S}_{i}^{\Delta}(i \in[4])$, there exists a $\delta>0$ such that for any $\zeta \in \mathbb{C}^{3}$ where $\left\|\zeta-\zeta_{0}\right\|_{\infty}<\delta$, we have

- $Z_{G}^{\sigma_{\Lambda}}(\zeta) \neq 0$ for every graph $G$ of degree at most $\Delta$ and every feasible configuration $\sigma_{\Lambda}$;

- the $\Delta$-bounded 2-spin system specified by $\zeta$ exhibits correlation decay.

Then via either Weitz's or Barvinok's algorithm, there is an FPTAS for computing $Z_{G}(\zeta)$.

Remark The choice of $\delta$ does not depend on the size of the graph, only on $\Delta$ and $\zeta_{0}$. In particular, let $D$ be a compact set in $\mathcal{S}_{i}^{\Delta}$ for some $i \in$ [4]. Then there is a uniform $\delta$ such that for all $\zeta$ in a complex neighborhood $D_{\delta}$ of radius $\delta$ around $D$, i.e., $\zeta \in D_{\delta}:=\{\mathbf{z} \in$ $\left.\mathbb{C}^{3} \mid\left\|\mathbf{z}-\mathbf{z}_{0}\right\|_{\infty}<\delta, \mathbf{z}_{0} \in D\right\}, Z_{G}(\zeta) \neq 0$ for every graph $G$ of degree at most $\Delta$. In addition, in order to apply Barvinok's algorithm, by Lemma 3.1, we need to make sure that the zero-free regions contain $\lambda=0$ (an easy computing point). This is true for $\mathcal{S}_{1}^{\Delta}, \mathcal{S}_{2}^{\Delta}$ and $\mathcal{S}_{3}^{\Delta}$. For parameters in $\mathcal{S}_{4}^{\Delta}$, we will reduce the problem to a case in $\mathcal{S}_{3}^{\Delta}$ by swapping $\beta$ and $\gamma$ and replacing $\lambda$ by $1 / \lambda$. Then, one can apply Barvinok's algorithm.

Acknowledgements We want to thank Jin-Yi Cai for many inspiring discussions and valuable comments on a preliminary version of this paper. Despite his support, he generously declined our invitation for co-authorship. We also want to thank Alex Scott, Jingcheng Liu and anonymous reviewers for their helpful comments.

Open Access This article is licensed under a Creative Commons Attribution 4.0 International License, which permits use, sharing, adaptation, distribution and reproduction in any medium or format, as long as you give appropriate credit to the original author(s) and the source, provide a link to the Creative Commons licence, and indicate if changes were made. The images or other third party material in this article are included in the article's Creative Commons licence, unless indicated otherwise in a credit line to the material. If material is not included in the article's Creative Commons licence and your intended use is not permitted by statutory regulation or exceeds the permitted use, you will need to obtain permission directly from the copyright holder. To view a copy of this licence, visit http://creativecommons.org/licenses/by/4.0/.

\section{A Appendix}

\section{A.1 Self-Avoiding Walk Tree}

Given a graph $G=(V, E)$ and a vertex $v \in V$, the SAW tree of $G$ at $v$ denoted by $T_{\mathrm{SAW}}(G, v)$ is a tree with root $v$ that enumerates all paths originating from $v$ in $G$. Additional vertices closing cycles of $G$ are added as leaves of the tree (see Fig. 3 for an example). Each vertex in $V$ of $G$ is mapped to some vertices in $V_{\text {SAW }}$ of $T_{\text {SAW }}(G, v)$. For leaves in $V_{\text {SAW }}$ that close cycles, a boundary condition is imposed. The imposed spin of such a leaf depends on whether the orientation of the cycle is from a lower indexed vertex to a higher indexed vertex or conversely (i.e., the leaf is imposed to + if the orientation of the cycle is from a lower indexed vertex to a higher indexed vertex, and to - otherwise), where the order of indices is arbitrarily chosen 


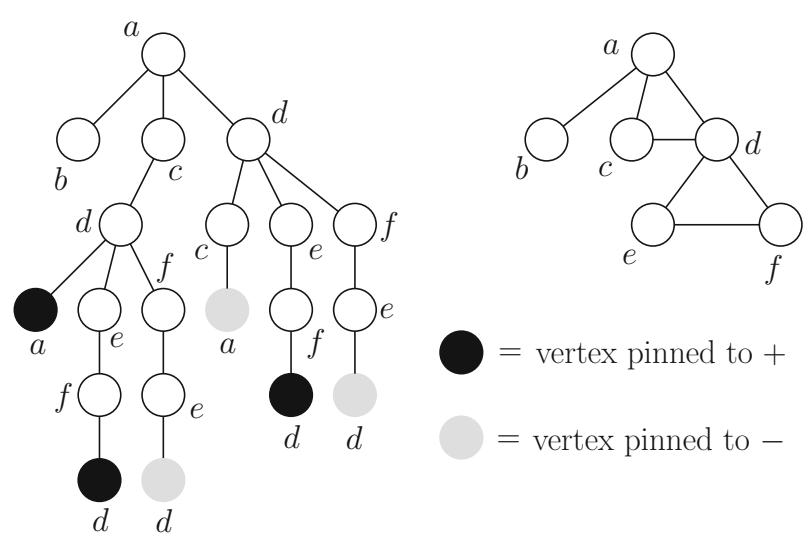

Fig. 3 Weitz's SAW tree construction

in $G$. Vertex sets $S \subseteq \Lambda \subseteq V$ are mapped to $S_{\mathrm{SAW}} \subseteq \Lambda_{\mathrm{SAW}} \subseteq V_{\mathrm{SAW}}$ respectively, and any configuration $\sigma_{\Lambda} \in\{+,-\}^{\Lambda}$ is mapped to a corresponding $\sigma_{\Lambda_{\mathrm{SAW}}} \in\{+,-\}^{\Lambda_{\mathrm{SAW}}}$. (For each vertex $v \in \Lambda_{\mathrm{SAW}}, \sigma_{\Lambda_{\mathrm{SAW}}}(v)$ takes the same assignment as its corresponding vertex in $\Lambda$ ).

Here is the key result for the SAW tree construction.

Theorem A.1 Let $G=(V, E)$ be a graph, $v \in V$, and $T=T_{\mathrm{SAW}}(G, v)$. Let $\sigma_{\Lambda} \in\{0,1\}^{\Lambda}$ be a configuration on $\Lambda \subseteq V$ where $v \notin \Lambda$, and $\sigma_{\Lambda_{\mathrm{SAW}}}$ be the corresponding configuration on $\Lambda_{\mathrm{SAW}} \subseteq V_{\mathrm{SAW}}$. Then,

1. $\operatorname{dist}_{G}(v, S)=\operatorname{dist}_{T}\left(v, S_{\mathrm{SAW}}\right)$ for any $S \subseteq V$, the maximum degree of $T$ is equal to the maximum degree of $G$, and the neighborhood of any vertex in $V_{\mathrm{SAW}}$ can be constructed in time proportional to the size of the neighborhood of the corresponding vertex in $V$.

2. The polynomial $Z_{G}^{\sigma_{\Lambda}}(\zeta)$ divides the polynomial $Z_{T}^{\sigma_{\Lambda} \mathrm{SAW}}(\zeta)$. In particular, if $\zeta_{0} \in \mathbb{C}^{3}$ is such that $Z_{T}^{\sigma_{\Lambda} \mathrm{SAW}}\left(\zeta_{0}\right) \neq 0$, then $Z_{G}^{\sigma_{\Lambda}}\left(\zeta_{0}\right) \neq 0$.

3. If $\zeta_{0} \in \mathbb{C}^{3}$ is such that $Z_{H}^{\tau_{\Lambda^{\prime}}}\left(\zeta_{0}\right) \neq 0$ for any graph $H$ and any feasible configuration $\tau_{\Lambda^{\prime}}$, then $R_{G, v}^{\sigma_{\Lambda}}\left(\zeta_{0}\right)=R_{T, v}^{\sigma_{\Lambda}} Z_{\text {AW }}\left(\zeta_{0}\right)$.

Proof The first part was proved by Weitz (Theorem 3.1 in [47]).

The second part was first proved for the hard-core model by Bencs [4] (although it was implicit in [47]). Later, it was proved for the Ising model by Liu, Sinclair, and Srivastava [30]. Following their proofs, one can directly check that this result holds for general 2-spin systems.

The third part was first proved for the hard-core model with positive external field $\lambda$ (i.e., $\beta=0, \gamma=1$ and $\lambda>0$ ) by Weitz [47]. Recently, it was proved for the Ising model with positive interaction parameter $\beta$ and nonzero complex external field $\lambda$ (i.e., $\beta=\gamma>0$ and $\lambda \in \mathbb{C} \backslash\{0\}$ ) by Peters and Regts [35]. Following the proof of Proposition 26 in [35], one can directly check that this result holds for general 2-spin systems with complex parameters $\zeta$. Note that the partition function $Z_{H}^{\tau_{\Lambda^{\prime}}}\left(\zeta_{0}\right) \neq 0$ for any graph $H$ and any feasible configuration $\tau_{\Lambda^{\prime}}$ ensures that the ratio $R_{H, v}^{\tau_{\Lambda^{\prime}}}\left(\zeta_{0}\right)$ is always well-defined for any graph $H$ and any feasible configuration $\tau_{\Lambda^{\prime}}$ where $v \notin \Lambda^{\prime}$.

\section{A.2 Proof of Lemma 2.7 for $\mathcal{S}_{1}^{\Delta}$ and $\mathcal{S}_{2}^{\Delta}$}

Lemma A.2 Fix $\Delta \geq 3$. For every $\zeta \in \mathcal{S}_{1}^{\Delta}$ or $\mathcal{S}_{2}^{\Delta}$, it satisfies real contraction for $\Delta$. 
The set $\mathcal{S}_{1}^{\Delta}$ was given in [48] and $\mathcal{S}_{2}^{\Delta}$ was given in [26]. The proofs of condition 2 of real contraction (bounding the 1-norm of the gradient $\nabla F_{\zeta, \mathbf{s}}^{\varphi}(\mathbf{x})$ ) for these two sets go back to [48] and [26] respectively. We include the proofs here for completeness.

Proof The case that $\lambda=0$ is trivial. We only consider the case that $\lambda \neq 0$.

Case 1: $\lambda \neq 0$ and $\zeta \in \mathcal{S}_{1}^{\Delta}$.

Let $r=\min \{1, \beta, 1 / \gamma\} \leq 1$ and $t=\max \{1, \beta, 1 / \gamma\} \geq 1$. We pick the interval $J=$ $\left[\lambda r^{\Delta-1}, \lambda t^{\Delta-1}\right]$ and the potential function $\varphi=\log (x)$ (this function is from [48]). Clearly, $\varphi$ is analytic on $J$ and $\varphi^{\prime}(x) \neq 0$ for all $x \in J$. Also, we know that $\lambda \in J,-\gamma \notin J$ and $-1 \notin J$ and $-1 \notin F_{\zeta, \mathbf{s}}\left(J^{k}\right)$ for every $\|\mathbf{s}\|_{1}=\Delta$. Since $\beta>0$ and $\gamma>0$, for any $x>0$, we have

$$
r \leq \min \{\beta, 1 / \gamma\} \leq \frac{\beta x+1}{x+\gamma} \leq \max \{\beta, 1 / \gamma\} \leq t .
$$

Thus, for any $\mathbf{x} \in J^{k}$, we have

$$
F_{\zeta, \mathbf{s}}(\mathbf{x})=\lambda \beta^{s_{1}} \gamma^{-s_{2}} \prod_{i=1}^{k}\left(\frac{\beta x_{i}+1}{x_{i}+\gamma}\right) \in\left[\lambda r^{\|\mathbf{s}\|_{1}}, \lambda t^{\|\mathbf{s}\|_{1}}\right] \subseteq\left[\lambda r^{\Delta-1}, \lambda t^{\Delta-1}\right] .
$$

Hence, $F_{\zeta, \mathbf{s}}\left(J^{k}\right) \subseteq J$ for every $\|\mathbf{s}\|_{1} \leq \Delta-1$.

Let $I=\varphi(J)$. Then, we consider the gradient $\nabla F_{\zeta, \mathbf{s}}^{\varphi}(\mathbf{x})$ for every $\|\mathbf{s}\|_{1} \leq \Delta-1$ and all $\mathbf{x} \in I^{k}$. We have

$$
F_{\zeta, \mathbf{s}}^{\varphi}(\mathbf{x})=\log \lambda+s_{1} \log \beta-s_{2} \log \gamma+\sum_{i=1}^{k} \log \left(\frac{\beta e^{x_{i}}+1}{e^{x_{i}}+\gamma}\right) .
$$

Thus, we have

$$
\left|\frac{\partial F_{\zeta, \mathrm{s}}^{\varphi}}{\partial x_{i}}\right|=\frac{|1-\beta \gamma|}{\beta e^{x_{i}}+\gamma e^{-x_{i}}+1+\beta \gamma} \leq \frac{|1-\beta \gamma|}{2 \sqrt{\beta \gamma}+1+\beta \gamma}=\frac{|1-\sqrt{\beta \gamma}|}{1+\sqrt{\beta \gamma}} .
$$

Here $\beta e^{x_{i}}+\gamma e^{-x_{i}} \geq 2 \sqrt{\beta \gamma}$ due to the AM-GM inequality. Since $\frac{\Delta-2}{\Delta}<\sqrt{\beta \gamma}<\frac{\Delta}{\Delta-2}$, we have $\frac{|1-\sqrt{\beta \gamma}|}{1+\sqrt{\beta \gamma}}<\frac{1}{\Delta-1}$. We can pick an positive $\eta$ such that $\left|\frac{\partial F_{\zeta, \mathrm{s}}^{\varphi}}{\partial x_{i}}\right| \leq \frac{1-\eta}{\Delta-1}$. Therefore, we have

$$
\left\|\nabla F_{\zeta, \mathbf{s}}^{\varphi}(\mathbf{x})\right\|_{1} \leq \sum_{i=1}^{k}\left|\frac{\partial F_{\zeta, \mathbf{s}}^{\varphi}}{\partial x_{i}}\right| \leq \frac{(1-\eta) k}{\Delta-1} \leq \frac{(1-\eta)\|\mathbf{s}\|_{1}}{\Delta-1} \leq 1-\eta
$$

for every $\|\mathbf{s}\|_{1} \leq \Delta-1$ and all $\mathbf{x} \in I^{k}$.

Case 2: $\lambda \neq 0$ and $\zeta \in \mathcal{S}_{2}^{\Delta}$.

If $\beta>0$, we still pick the same interval $J$ as in Case 1 . We know that condition 1 of real contraction is satisfied.

For the case that $\beta=0$, we pick the interval $J=[\ell, m]$, where $m=\max \left\{\lambda, \lambda / \gamma^{\Delta-1}\right\}>0$ and $\ell=\min \left\{\lambda, \lambda /(m+\gamma)^{\Delta-1}\right\}>0$. Clearly, we have $\lambda \in J,-\gamma \notin J,-1 \notin J$, and $-1 \notin F_{\zeta, \mathbf{s}}\left(J^{k}\right)$ for every $\|\mathbf{s}\|_{1}=\Delta$. Recall that when $\beta=0$, we only consider recursion functions $F_{\zeta, \mathbf{s}}$ where $s_{1}=0$. Then for every $\mathbf{s}$ where $\|\mathbf{s}\|_{1} \leq \Delta-1$ and $s_{1}=0$ and all $\mathbf{x} \in J^{k}$, we have

$$
\ell \leq \frac{\lambda}{(m+\gamma)^{s_{2}+k}} \leq F_{\zeta, \mathbf{s}}(\mathbf{x})=\lambda \gamma^{-s_{2}} \prod_{i=1}^{k}\left(\frac{1}{x_{i}+\gamma}\right) \leq \frac{\lambda}{\gamma^{s_{2}+k}} \leq m .
$$


Hence $F_{\zeta, \mathbf{s}}\left(J^{k}\right) \subseteq J$ for every $\|\mathbf{s}\|_{1} \leq \Delta-1$.

Now, we pick the potential function

$$
\varphi(x)=\int_{1}^{x} \frac{1}{\sqrt{y(\beta y+1)(y+\gamma)}} d y
$$

which is the one from [26]. Clearly, $\varphi$ is analytic on $J$ and $\varphi^{\prime}(x) \neq 0$ for all $x \in J$. We consider the gradient $\nabla F_{\zeta, \mathbf{s}}^{\varphi}(\mathbf{x})$. By calculation, we have $\left\|\nabla F_{\zeta, \mathbf{s}}^{\varphi}(\mathbf{x})\right\|_{1}=H_{\zeta, \mathbf{s}}\left(\varphi^{-1}(\mathbf{x})\right)$ where $\boldsymbol{\varphi}^{-1}(\mathbf{x})=\left(\varphi^{-1}\left(x_{1}\right), \ldots, \varphi^{-1}\left(x_{k}\right)\right)$ and

$$
H_{\zeta, \mathbf{s}}(\mathbf{x})=(1-\beta \gamma) \cdot \sqrt{\frac{F_{\zeta, \mathbf{s}}(\mathbf{x})}{\left(\beta F_{\zeta, \mathbf{s}}(\mathbf{x})+1\right)\left(F_{\zeta, \mathbf{s}}(\mathbf{x})+\gamma\right)}} \cdot \sum_{i=1}^{k} \sqrt{\frac{x_{i}}{\left(\beta x_{i}+1\right)\left(x_{i}+\gamma\right)}} .
$$

We want to bound $\left\|\nabla F_{\zeta, \mathbf{s}}^{\varphi}(\mathbf{x})\right\|_{1}$ for every $\|\mathbf{s}\|_{1} \leq \Delta-1$ and all $\mathbf{x} \in I^{k}$ where $I=\varphi(J)$, which is equivalent to bound $H_{\zeta, \mathbf{s}}\left(\varphi^{-1}(\mathbf{x})\right)$ for all $\varphi^{-1}(\mathbf{x}) \in J^{k} \subseteq \mathbb{R}_{+}^{k}$. We will show that there exists some $\eta>0$ such that $H_{\zeta, \mathbf{s}}(\mathbf{x}) \leq 1-\eta$ for every $\|\mathbf{s}\|_{1} \leq \Delta-1$ and all $\mathbf{x} \in \mathbb{R}_{+}^{k}$. This will finish the proof. Note that for any $\mathbf{s}=\left(s_{1}, s_{2}, k\right)$ with $k=0$, we have $\nabla F_{\zeta, \mathbf{s}}^{\varphi}(\mathbf{x}) \equiv 0$ and we are done. Thus, we may assume that $k \geq 1$. We prove our claim in two steps.

Step 1. Let

$$
h_{d}(x)=d(1-\beta \gamma) \cdot \sqrt{\frac{x}{(\beta x+1)(x+\gamma)}} \cdot \sqrt{\frac{\lambda\left(\frac{\beta x+1}{x+\gamma}\right)^{d}}{\left(\beta \lambda\left(\frac{\beta x+1}{x+\gamma}\right)^{d}+1\right)\left(\lambda\left(\frac{\beta x+1}{x+\gamma}\right)^{d}+\gamma\right)}}
$$

be the symmetrized univariate version of $H_{\zeta, \mathbf{s}}(\mathbf{x})$ where $d=\|\mathbf{s}\|_{1} \leq \Delta-1$. We show that there exists some $\hat{x}>0$ such that $H_{\zeta, \mathbf{s}}(\mathbf{x}) \leq h_{d}(\hat{x})$.

Consider some $\mathbf{x}=\left(x_{1}, \ldots, x_{k}\right) \in \mathbb{R}_{+}^{k}$. For every $x_{i}>0$, let $z_{i}=\frac{\beta x_{i}+1}{x_{i}+\gamma}$, and we have $z_{i} \in\left(\beta, \frac{1}{\gamma}\right)$. Also, let $z_{k+1}=\cdots=z_{k+s_{1}}=\beta$ and $z_{k+s_{1}+1}=\cdots=z_{d}=\frac{1}{\gamma}$. Then, we have

$$
H_{\zeta, \mathbf{s}}(\mathbf{x})=\sqrt{\frac{\lambda \prod_{i=1}^{d} z_{i}}{\left(\beta \cdot \lambda \prod_{i=1}^{d} z_{i}+1\right)\left(\lambda \prod_{i=1}^{d} z_{i}+\gamma\right)}} \cdot \sum_{i=1}^{d} \sqrt{\left(\frac{1}{z_{i}}-\gamma\right)\left(z_{i}-\beta\right) .}
$$

By Cauchy-Schwarz inequality and AM-GM inequality, we have

$$
\begin{aligned}
\sum_{i=1}^{d} \sqrt{\left(\frac{1}{z_{i}}-\gamma\right)\left(z_{i}-\beta\right)} & \leq d \sqrt{1+\beta \gamma-\frac{1}{d} \sum_{i=1}^{d}\left(\gamma z_{i}+\frac{\beta}{z_{i}}\right)} \\
& \leq d \sqrt{1+\beta \gamma-\gamma\left(\prod_{i=1}^{d} z_{i}\right)^{\frac{1}{d}}-\beta\left(\prod_{i=1}^{d} z_{i}\right)^{-\frac{1}{d}}} .
\end{aligned}
$$


Let $\hat{z}=\left(\prod_{i=1}^{d} z_{i}\right)^{\frac{1}{d}}$. Since $z_{i} \in\left(\beta, \frac{1}{\gamma}\right)$ for $i \in[k]$ and $k \geq 1$, we know $\hat{z} \in\left(\beta, \frac{1}{\gamma}\right)$. Then, we have

$$
H_{\zeta, \mathbf{s}}(\mathbf{x}) \leq \sqrt{\frac{\lambda \hat{z}^{d}}{\left(\beta \lambda \hat{z}^{d}+1\right)\left(\lambda \hat{z}^{d}+\gamma\right)}} \cdot d \sqrt{1+\beta \gamma-\gamma \hat{z}-\frac{\beta}{\hat{z}}}=d \sqrt{\frac{\lambda \hat{z}^{d}\left(\frac{1}{\hat{z}}-\gamma\right)(\hat{z}-\beta)}{\left(\beta \lambda \hat{z}^{d}+1\right)\left(\lambda \hat{z}^{d}+\gamma\right)}} .
$$

Let $\hat{x}=\frac{1-\gamma \hat{z}}{\hat{z}-\beta}$. Then we have $\hat{x}>0$, and

$$
\begin{aligned}
H_{\zeta, \mathbf{s}}(\mathbf{x}) & \leq d(1-\beta \gamma) \cdot \sqrt{\frac{\hat{x}}{(\beta \hat{x}+1)(\hat{x}+\gamma)}} \cdot \sqrt{\frac{\lambda\left(\frac{\beta \hat{x}+1}{\hat{x}+\gamma}\right)^{d}}{\left(\beta \lambda\left(\frac{\beta \hat{x}+1}{\hat{x}+\gamma}\right)^{d}+1\right)\left(\lambda\left(\frac{\beta \hat{x}+1}{\hat{x}+\gamma}\right)^{d}+\gamma\right)}} \\
& =
\end{aligned}
$$

Step 2. We show that there exists some $\eta>0$ such that $h_{d}(x) \leq 1-\eta$ for every $1 \leq d \leq \Delta-1$ and all $x>0$.

We characterize the point $x$ at which $h_{d}(x)$ achieves its maximum. Recall that we define $f_{d}(x)=\lambda\left(\frac{\beta x+1}{x+\gamma}\right)^{d}$ and we have $f_{d}^{\prime}(x)=\frac{d(\beta \gamma-1) f_{d}(x)}{(\beta x+1)(x+\gamma)}$. Consider the derivative of $h_{d}(x)$, we have

$$
h_{d}^{\prime}(x)=\frac{d(1-\beta \gamma) g_{d}^{\prime}(x)}{2 \sqrt{g_{d}(x)}},
$$

where

$$
g_{d}(x)=\frac{x f_{d}(x)}{(\beta x+1)(x+\gamma)\left(\beta f_{d}(x)+1\right)\left(f_{d}(x)+\gamma\right)},
$$

and its derivative

$$
\begin{aligned}
g_{d}^{\prime}(x)= & \frac{d(1-\beta \gamma) x f_{d}(x)}{(\beta x+1)^{2}(x+\gamma)^{2}\left(\beta f_{d}(x)+1\right)\left(f_{d}(x)+\gamma\right)} . \\
& \left(\frac{\gamma-\beta x^{2}}{d(1-\beta \gamma) x}-\frac{\gamma-\beta f_{d}(x)^{2}}{\left(\beta f_{d}(x)+1\right)\left(f_{d}(x)+\gamma\right)}\right) .
\end{aligned}
$$

We want to solve $h_{d}^{\prime}(x)=0$. Since $1-\beta \gamma>0$, it is equivalent to solve the equation

$$
\frac{\gamma-\beta x^{2}}{d(1-\beta \gamma) x}=\frac{\gamma-\beta f_{d}(x)^{2}}{\left(\beta f_{d}(x)+1\right)\left(f_{d}(x)+\gamma\right)} \text {. }
$$

Note that as $x$ increases from 0 to $+\infty$, the function $\frac{\gamma-\beta x^{2}}{d(1-\beta \gamma) x}$ strictly decreases from $+\infty$ to $-\infty$. On the other hand, the function $\frac{\gamma-\beta f_{d}(x)^{2}}{\left(\beta f_{d}(x)+1\right)\left(f_{d}(x)+\gamma\right)}$ strictly increases since $f_{d}(x)$ strictly decreases as $x$ increases. Therefore equation (1) has a unique solution in $(0,+\infty)$, denoted by $x_{d}$. Furthermore, we have

$$
g_{d}^{\prime}(x) \begin{cases}>0 & \text { if } 0<x<x_{d} \\ =0 & \text { if } x=x_{d} \\ <0 & \text { if } x>x_{d}\end{cases}
$$


Clearly the sign of $h_{d}^{\prime}(x)$ is the same as that of $g_{d}^{\prime}(x)$. Hence $h_{d}(x)$ achieves its maximum when $x=x_{d}$. Then for any $x>0$, we have

$$
\begin{aligned}
h_{d}(x) \leq h_{d}\left(x_{d}\right) & =d(1-\beta \gamma) \cdot \sqrt{\frac{x_{d} f_{d}\left(x_{d}\right)}{\left(\beta x_{d}+1\right)\left(x_{d}+\gamma\right)\left(\beta f_{d}\left(x_{d}\right)+1\right)\left(f_{d}\left(x_{d}\right)+\gamma\right)}} \\
& =\sqrt{\frac{d(1-\beta \gamma) f_{d}\left(x_{d}\right)\left(\gamma-\beta x_{d}^{2}\right)}{\left(\beta x_{d}+1\right)\left(x_{d}+\gamma\right)\left(\gamma-f_{d}\left(x_{d}\right)^{2}\right)}} .
\end{aligned}
$$

Here, we substitute $\left(\beta f_{d}\left(x_{d}\right)+1\right)\left(f_{d}\left(x_{d}\right)+\gamma\right)$ by $\frac{d(1-\beta \gamma)\left(\gamma-\beta f_{d}\left(x_{d}\right)^{2}\right) x_{d}}{\gamma-\beta x_{d}^{2}}$ according to (1). Consider the function

$$
p_{d}(x):=\sqrt{\frac{d(1-\beta \gamma) f_{d}(x)\left(\gamma-\beta x^{2}\right)}{(\beta x+1)(x+\gamma)\left(\gamma-f_{d}(x)^{2}\right)}} .
$$

Then, we have $h_{d}(x) \leq p_{d}\left(x_{d}\right)$ for any $x>0$. Now, we claim that for any $1 \leq d \leq \Delta-1$,

$$
p_{d}\left(x_{d}\right) \leq p_{d}\left(\hat{x}_{d}\right)
$$

where $\hat{x}_{d}$ is the unique positive fixed point of $f_{d}(x)$. To prove the above claim, we only need to show that $p_{d}(x)$ is decreasing if $\hat{x}_{d} \leq x_{d}$ and increasing if $\hat{x}_{d}>x_{d}$.

- If $\hat{x}_{d} \leq x_{d}$, we will show that $p_{d}(x)$ is decreasing on the range $\left[\hat{x}_{d}, x_{d}\right]$. By (2), we know $g_{d}^{\prime}\left(\hat{x}_{d}\right) \geq 0$. Note that

$$
g_{d}^{\prime}\left(\hat{x}_{d}\right)=\frac{d(1-\beta \gamma)\left(\gamma-\beta \hat{x}_{d}^{2}\right) \hat{x}_{d}^{2}}{\left(\beta \hat{x}_{d}+1\right)^{3}\left(\hat{x}_{d}+\gamma\right)^{3}} \cdot\left(\frac{1}{d(1-\beta \gamma) \hat{x}_{d}}-\frac{1}{\left(\beta \hat{x}_{d}+1\right)\left(\hat{x}_{d}+\gamma\right)}\right) .
$$

Since $\zeta$ is up-to- $\Delta$ unique, we have $\left|f_{d}^{\prime}\left(\hat{x}_{d}\right)\right|=\frac{d(1-\beta \gamma) \hat{x}_{d}}{\left(\beta \hat{x}_{d}+1\right)\left(\hat{x}_{d}+\gamma\right)}<1$ and hence $\frac{1}{d(1-\beta \gamma) \hat{x}_{d}}-$ $\frac{1}{\left(\beta \hat{x}_{d}+1\right)\left(\hat{x}_{d}+\gamma\right)}>0$. Thus, we have $\gamma-\beta \hat{x}_{d}^{2} \geq 0$. Also, since $f_{d}(x)$ strictly decreases as $x$ increases, we have

$$
\gamma-\beta f_{d}\left(x_{d}\right)^{2} \geq \gamma-\beta f_{d}\left(\hat{x}_{d}\right)^{2}=\gamma-\beta \hat{x}_{d}^{2} \geq 0 .
$$

Then by equality (1), $\gamma-\beta x_{d}^{2}$ and $\gamma-\beta f_{d}\left(x_{d}\right)^{2}$ must be both positive or negative. Thus we have $\gamma-\beta x_{d}^{2} \geq 0$. Then both $\frac{\gamma-\beta x^{2}}{(\beta x+1)(x+\gamma)}$ and $\frac{f_{d}(x)}{\gamma-\beta f_{d}(x)^{2}}$ are positive and strictly decreasing on $\left[\hat{x}_{d}, x_{d}\right]$. Thus, $p_{d}(x)$ is strictly decreasing on $\left[\hat{x}_{d}, x_{d}\right]$, and hence $p_{d}\left(x_{d}\right) \leq p_{d}\left(\hat{x}_{d}\right)$.

- Otherwise, $\hat{x}_{d}>x_{d}$. By a similar argument as the above, we have $\gamma-\beta f_{d}\left(\hat{x}_{d}\right)^{2}=\gamma-$ $\beta{\hat{x_{d}}}^{2}<0, \gamma-\beta f_{d}\left(x_{d}\right)^{2}<0$ and $\gamma-\beta x_{d}^{2}<0$. Hence both $\frac{\gamma-\beta x^{2}}{(\beta x+1)(x+\gamma)}$ and $\frac{f_{d}(x)}{\gamma-\beta f_{d}(x)^{2}}$ are negative and strictly decreasing on $\left[x_{d}, \hat{x}_{d}\right]$ and hence their product is positive and increasing on $\left[x_{d}, \tilde{x}_{d}\right]$. Thus we have $p_{d}(x)$ is increasing, and $p_{d}\left(x_{d}\right) \leq p_{d}\left(\hat{x}_{d}\right)$.

Combining (3) and (4), we have for all $x>0$,

$$
h_{d}(x) \leq h_{d}\left(x_{d}\right)=p_{d}\left(x_{d}\right) \leq p_{d}\left(\hat{x}_{d}\right)=\sqrt{\frac{d(1-\beta \gamma) \hat{x}_{d}}{\left(\beta \hat{x}_{d}+1\right)\left(\hat{x}_{d}+\gamma\right)}}=\sqrt{\left|f_{d}^{\prime}\left(\hat{x}_{d}\right)\right|} .
$$

Since $\zeta$ is up-to- $\Delta$ unique, there exists a constant $0<c<1$ such that $\left|f_{d}^{\prime}\left(\hat{x}_{d}\right)\right| \leq c$ for every integer $1 \leq d \leq \Delta-1$. Let $\eta=1-\sqrt{c}>0$. Then, we have $h_{d}(x) \leq 1-\eta$ for all $x>0$. 


\section{References}

1. Asano, T.: Lee-Yang theorem and the Griffiths inequality for the anisotropic Heisenberg ferromagnet. Phys. Rev. Lett. 24(25), 1409 (1970)

2. Barahona, F.: On the computational complexity of Ising spin glass models. J. Phys. A 15(10), 3241 (1982)

3. Barvinok, A.: Combinatorics and Complexity of Partition Functions, vol. 9. Springer, New York (2016)

4. Bencs, F.: On trees with real-rooted independence polynomial. Discret. Math. 341(12), 3321-3330 (2018)

5. Bencs, F. , Csikvári, P.: Note on the zero-free region of the hard-core model. arXiv preprint arXiv:1807.08963 (2018)

6. Bulatov, A.: The complexity of the counting constraint satisfaction problem. J. ACM (JACM) 60(5), 1-41 (2013)

7. Bulatov, A., Grohe, M.: The complexity of partition functions. Theor. Comput. Sci. 348(2-3), 148-186 (2005)

8. Cai, J.-Y., Chen, X.: Complexity of counting CSP with complex weights. J. ACM (JACM) 64(3), 1-39 (2017)

9. Cai, J.-Yi., Chen, X., Pinyan, L.: Graph homomorphisms with complex values: A dichotomy theorem. SIAM J. Comput. 42(3), 924-1029 (2013)

10. Cai, J.-Y., Pinyan, L., Xia, M.: The complexity of complex weighted Boolean \#CSP. J. Comput. Syst. Sci. 80(1), 217-236 (2014)

11. Dobrushin, R.L., Shlosman, S.B.: Completely analytical Gibbs fields. In: Statistical Physics and Dynamical Systems, pp. 371-403. Springer, Berlin (1985)

12. Dobrushin, R.L., Shlosman, S.B.: Completely analytical interactions: constructive description. J. Stat. Phys. 46(5), 983-1014 (1987)

13. Dyer, M., Goldberg, L.A., Jerrum, M.: The complexity of weighted Boolean \#CSP. SIAM J. Comput. 38(5), 1970-1986 (2009)

14. Dyer, M., Greenhill, C.: The complexity of counting graph homomorphisms. Random Struct. Algorithms 17(3-4), 260-289 (2000)

15. Dyer, M., Richerby, D.: An effective dichotomy for the counting constraint satisfaction problem. SIAM J. Comput. 42(3), 1245-1274 (2013)

16. Galanis, A., Štefankovič, D., Vigoda, E.: Inapproximability of the partition function for the antiferromagnetic Ising and hard-core models. Comb. Probab. Comput. 25(4), 500-559 (2016)

17. Grohe, M., Jerrum, M., Thurley, M., Leslie Ann Goldberg: A complexity dichotomy for partition functions with mixed signs. SIAM J. Comput. 39(7), 3336-3402 (2010)

18. Goldberg, L.A., Jerrum, M., Paterson, M.: The computational complexity of two-state spin systems. Random Struct. Algorithms 23(2), 133-154 (2003)

19. Guo, H., Pinyan, L.: Zeros of ferromagnetic 2-spin systems. In Proceedings of the Fourteenth Annual ACM-SIAM Symposium on Discrete Algorithms, pp. 181-192. SIAM (2020)

20. Guo, H., Pinyan, L.: Uniqueness, spatial mixing, and approximation for ferromagnetic 2-spin systems. ACM Trans. Comput. Theory (TOCT) 10(4), 1-25 (2018)

21. Harvey, N., Srivastava, P., Vondrák, J.: Computing the independence polynomial: from the tree threshold down to the roots. In Proceedings of the Twenty-Ninth Annual ACM-SIAM Symposium on Discrete Algorithms, pp. 1557-1576. SIAM (2018)

22. Jerrum, M., Sinclair, A.: Polynomial-time approximation algorithms for the Ising model. SIAM J. Comput. 22(5), 1087-1116 (1993)

23. Jerrum, M., Valiant, L., Vazirani, V.: Random generation of combinatorial structures from a uniform distribution. Theor. Comput. Sci. 43, 169-188 (1986)

24. Lee, T.-D., Yang, -N.: Statistical theory of equations of state and phase transitions. II. Lattice gas and Ising model. Phys. Rev. 87, 410-419 (1952)

25. Li, L., Pinyan, L., Yin, Y.: Approximate counting via correlation decay in spin systems. In Proceedings of the twenty-third annual ACM-SIAM symposium on Discrete Algorithms, pp. 922-940. SIAM (2012)

26. Li, L., Lu, P., Yin, Y.: Correlation decay up to uniqueness in spin systems. In Proceedings of the twentyfourth annual ACM-SIAM symposium on Discrete algorithms, pp. 67-84. SIAM (2013)

27. Lieb, E., Sokal, A.: A general Lee-Yang theorem for one-component and multicomponent ferromagnets. Commun. Math. Phys. 80(2), 153-179 (1981)

28. Liu, J.: Approximate counting, phase transitions and geometry of polynomials. PhD thesis, UC Berkeley (2019)

29. Liu, J., Lu, P., Zhang, C.: The complexity of ferromagnetic two-spin systems with external fields. arXiv preprint arXiv:1402.4346 (2014)

30. Liu, J.: Sinclair, Alistair, Srivastava, Piyush: Fisher zeros and correlation decay in the Ising model. J. Math. Phys. 60(10), 103304 (2019) 
31. Liu, J., Sinclair, A., Srivastava, P.: The Ising partition function: zeros and deterministic approximation. J. Stat. Phys. 174(2), 287-315 (2019)

32. Newman, C.: Zeros of the partition function for generalized Ising systems. Commun. Pure Appl. Math. 27(2), 143-159 (1974)

33. Patel, V., Regts, G.: Deterministic polynomial-time approximation algorithms for partition functions and graph polynomials. SIAM J. Comput. 46(6), 1893-1919 (2017)

34. Peters, H., Regts, G.: On a conjecture of Sokal concerning roots of the independence polynomial. Mich. Math. J. 68(1), 33-55 (2019)

35. Peters, H., Regts, G.: Location of zeros for the partition function of the Ising model on bounded degree graphs. J. Lond. Math. Soc. 101(2), 765-785 (2020)

36. Restrepo, R., Shin, J., Tetali, P., Vigoda, E., Yang, L.: Improved mixing condition on the grid for counting and sampling independent sets. Probab. Theory Relat. Fields 156(1-2), 75-99 (2013)

37. Ruelle, D.: Extension of the Lee-Yang circle theorem. Phys. Rev. Lett. 26(6), 303 (1971)

38. Scott, A., Sokal, A.: The repulsive lattice gas, the independent-set polynomial, and the Lovász local lemma. J. Stat. Phys. 118(5-6), 1151-1261 (2005)

39. Shao, S., Sun, Y.: Contraction: a unified perspective of correlation decay and zero-freeness of 2-spin systems. arXiv preprint arXiv:1909.04244 (2019)

40. Simon, B., GriffithsGriffiths, R.B.: The $\left(\phi^{4}\right)_{2}$ field theory as a classical Ising model. Commun. Math. Phys. 33(2), 145-164 (1973)

41. Sinclair, A., Srivastava, P., Štefankovič, D., Yin, Y.: Spatial mixing and the connective constant: optimal bounds. Probab. Theory Relat. Fields 168(1-2), 153-197 (2017)

42. Sinclair, A., Srivastava, P., Thurley, M.: Approximation algorithms for two-state anti-ferromagnetic spin systems on bounded degree graphs. J. Stat. Phys. 155(4), 666-686 (2014)

43. Sly, A., Sun, N.: Counting in two-spin models on d-regular graphs. Ann. Probab. 42(6), 2383-2416 (2014)

44. Sokal, A.: A personal list of unsolved problems concerning lattice gases and antiferromagnetic potts models. arXiv preprint arXiv:cond-mat/0004231 (2000)

45. Sokal, A.: Bounds on the complex zeros of (di)chromatic polynomials and Potts-model partition functions. Comb. Probab. Comput. 10(1), 41-77 (2001)

46. Stein, El., Shakarchi, R.: Complex Analysis, vol. 2. Princeton University Press, Princeton (2010)

47. Weitz, D.: Counting independent sets up to the tree threshold. In Proceedings of the thirty-eighth annual ACM symposium on Theory of computing, pp. 140-149 (2006)

48. Zhang, J., Liang, H., Bai, F.: Approximating partition functions of the two-state spin system. Inf. Process. Lett. 111(14), 702-710 (2011)

Publisher's Note Springer Nature remains neutral with regard to jurisdictional claims in published maps and institutional affiliations. 